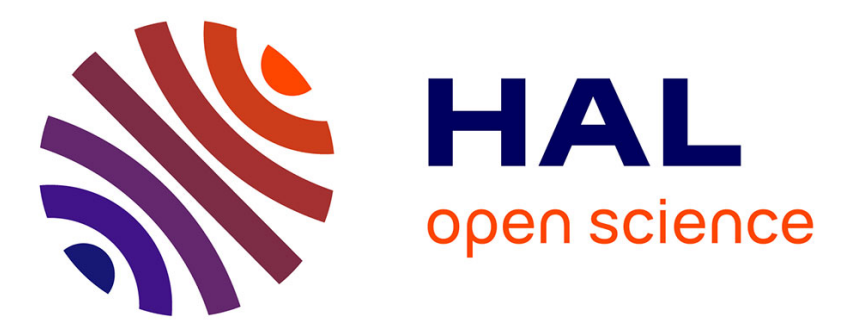

\title{
Multiscale computational homogenization of heterogeneous shells at small strains with extensions to finite displacements and buckling
}

Yu Cong, Saeid Nezamabadi, Hamid Zahrouni, Julien Yvonnet

\section{- To cite this version:}

Yu Cong, Saeid Nezamabadi, Hamid Zahrouni, Julien Yvonnet. Multiscale computational homogenization of heterogeneous shells at small strains with extensions to finite displacements and buckling. International Journal for Numerical Methods in Engineering, 2015, 104 (4), pp.236-259. 10.1002/nme.4927 . hal-01165988

\section{HAL Id: hal-01165988 \\ https://hal.science/hal-01165988}

Submitted on 10 Mar 2016

HAL is a multi-disciplinary open access archive for the deposit and dissemination of scientific research documents, whether they are published or not. The documents may come from teaching and research institutions in France or abroad, or from public or private research centers.
L'archive ouverte pluridisciplinaire HAL, est destinée au dépôt et à la diffusion de documents scientifiques de niveau recherche, publiés ou non, émanant des établissements d'enseignement et de recherche français ou étrangers, des laboratoires publics ou privés. 


\title{
Multiscale computational homogenization of heterogeneous shells at small strains with extensions to finite displacements and buckling
}

\author{
Yu Cong ${ }^{1, *, \dagger}$, Saeid Nezamabadi ${ }^{2},{\text { Hamid } Z^{2} \text { ahrouni }}^{1}$ and Julien Yvonnet $^{3}$ \\ ${ }^{1}$ Laboratoire d'Étude des Microstructures et de Mécanique des Matériaux, Université de Lorraine, UMR CNRS 7239, \\ Ile du Saulcy, F-57045 Metz Cedex 01, France \\ ${ }^{2}$ Laboratoire de Mécanique et Génie Civil, Université Montpellier 2, UMR CNRS 5508, CC048 Place Eugène \\ Bataillon, 34095 Montpellier Cedex 05, France \\ ${ }^{3}$ Laboratoire Modélisation et Simulation Multi Échelle, Université Paris-Est, UMR CNRS 8208, 5 Bd Descartes, \\ 77454 Marne-la-Vallée Cedex 02, France
}

\begin{abstract}
SUMMARY
In this paper, a framework for computational homogenization of shell structures is proposed in the context of small-strain elastostatics, with extensions to large displacements and large rotations. At the macroscopic scale, heterogeneous thin structures are modeled using a homogenized shell model, based on a versatile three-dimensional seven-parameter shell formulation, incorporating a through-thickness and pre-integrated constitutive relationship. In the context of small strains, we show that the local solution on the elementary cell can be decomposed into six strains and six-strain gradient modes, associated with corresponding boundary conditions. The heterogeneities can have arbitrary morphology but are assumed to be periodically distributed in the tangential direction of the shell. We then propose an extension of the small-strain framework to geometrical nonlinearities. The procedure is purely sequential and does not involve coupling between scales. The homogenization method is validated and illustrated through examples involving large displacements and buckling of heterogeneous plates and shells.
\end{abstract}

KEY WORDS: computational homogenization; shells; thin structures; heterogeneous materials; multiscale methods

\section{INTRODUCTION}

Heterogeneous thin plates and shells are widely used in modern applications that require unconventional mechanical and thermal performances. Such structures include among others a wide range of composite thin sheets and panels that are increasingly applied in aerospace and automotive industries, where the need for enhanced lightness and strength is continuously growing. Modeling heterogeneous thin structures requires homogenized models, to avoid the costly computations associated with an explicit description of all heterogeneities.

Theoretical research for treating laminated thin structures has been widely developed (e.g., [1-5]). Depending on theories of laminated plates and shells, these methods are mainly valid for classical multilayer structures and do not account for complex multiphase morphologies. For thin structures composed of unconventional microstructures, many authors proposed homogenized models based on investigation of the geometrical pattern of the core structure. This is the case in the work of Abbes and Guo [6] and of Talbi [7]. Both of them focused on modeling thin-plate structures with corrugated core geometries based on analytical analysis of the core pattern. A similar approach has

*Correspondence to: Yu Cong, Laboratoire d'Étude des Microstructures et de Mécanique des Matériaux, Université de Lorraine, UMR CNRS 7239, Ile du Saulcy, F-57045 Metz Cedex 01, France. †E-mail: yyc@esi-group.com 
been adopted in the work of Lebée and Sab [8-10] who proposed a thick-plate model for a special kind of sandwich plate with periodic chevron folded core. Most of these techniques strongly rely on analytical or semi-analytical analysis of the structure's specific geometry pattern, thus may appear limited for general applications involving arbitrary microstructures.

In the aim of developing a generalized multiscale framework that does not rely on the geometrical specificity of the microstructure, most attention has been turned to developing computational multiscale approaches. In a series of works, Cartraud et al., focused on computational homogenization of slender periodic structures based on asymptotic expansion methods, where beams with sinusoidal microstructures (Cartraud and Messager [11]) and plates with corrugated cores (Buannic, Cartraud, and Quesnel [12]) have been studied in the context of Kirchhoff-Love and Reinssler-Mindlin plate models. Extending previous works on computational homogenization for bulk materials (e.g., [13-18] ), Coenen et al. proposed a homogenization framework for nonlinear thin structures [19], involving a concurrent computational methodology where nonlinear computations are required at all integration points of the macroscopic shell model. In this work, the authors proposed to describe the thin structure's macroscopic kinematics in terms of an in-plane deformation gradient tensor and a curvature tensor. With an appropriate definition of the scale transition based on average of both the resultant stress and moment, heterogeneous thin structures were homogenized towards a KirchhoffLove plate model. In a recent work, Helfen and Diebels [20] introduced a general homogenization framework based on a plate model allowing for thickness changes.

Several drawbacks are encountered in the mentioned works: (i) most of them are restricted to plate theories and do not take into account thin structures with curved surfaces, which are of major interest for industrial applications; (ii) the analytical methods are restricted to some specific classes of microstructural patterns; (iii) the computational multiscale methods often rely on coupled calculations at both scales [19], which induce high computational costs; and (iv) the proposed numerical techniques do not offer extended capability to deal with problems involving instabilities, such as buckling.

In the present work, a computational homogenization procedure is developed in the context of heterogeneous shells, able to take into account arbitrary microstructures periodically distributed in the tangential directions to the shells, without coupled computations at both scales, and able to deal with geometrical nonlinearities, thus allowing to study buckling phenomena.

The outline of the paper is as follows. In Section 2, the kinematic description of the sevenparameter shell model is reviewed. In Section 3, the homogenization methodology is developed in the context of small strains and extended in section 4 to geometrical nonlinearities. Finally, the method is illustrated and validated through several examples in Section 5. To make the paper selfcontained, a review of practical finite element implementation of the shell model is provided in Appendices A and B.

\section{SHELL KINEMATICS AT SMALL STRAINS}

\subsection{Preliminary definitions}

At the macroscopic scale, we consider a versatile seven-parameter shell model with enhanced assumed strain enrichment (e.g., [21-24]). The associated kinematics in the context of small strains are reviewed in the following. This shell formulation accounts for transverse normal and shear deformations and does not have the thickness 'locking' issue, which consistently arises for classical shell models when dealing with bending dominated kinematics.

Basic kinematics of the shell continuum is represented in Figure 1, in which trajectory of an arbitrary material point can be described considering its position vectors $\mathbf{X}$ and $\mathbf{x}$, respectively, in the reference and current configurations. Although expressed in the global coordinate system, both vectors can be located with the local curvilinear coordinates $\left(\theta^{1}, \theta^{2}, \theta^{3}\right)$, for which $\theta^{1}$ and $\theta^{2}$ lie in the mid-surface of the shell continuum and $\theta^{3}$ in the transversal direction with respect to the midsurface. In the reference configuration, the position vector $\mathbf{X}$ of an arbitrary material point can be defined as

$$
\mathbf{X}\left(\theta^{\alpha}, \theta^{3}\right)=\mathbf{R}\left(\theta^{\alpha}\right)+\theta^{3} \mathbf{a}_{3}\left(\theta^{\alpha}\right)
$$




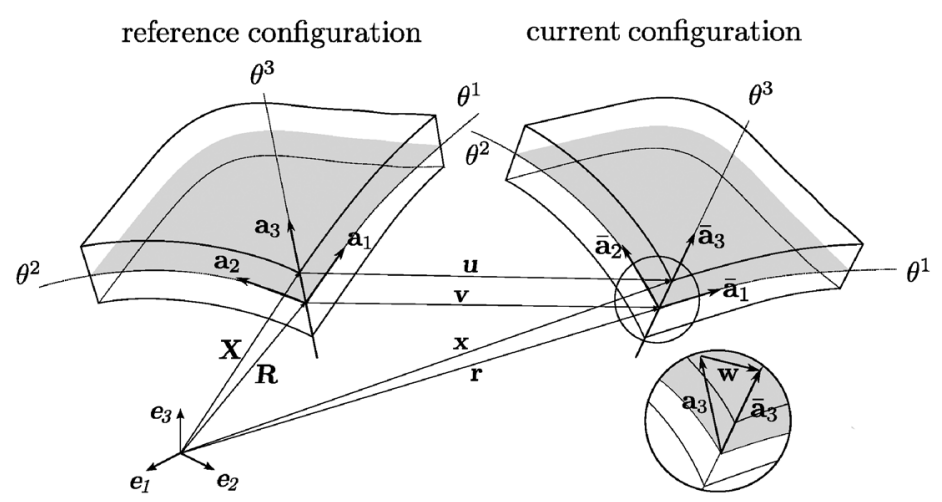

Figure 1. Representation of 3D shell geometry and its kinematics.

where $\mathbf{R}\left(\theta^{\alpha}\right)(\alpha=1,2)$ refers to its projection point on the mid-surface $\left(\theta^{3}=0\right)$ and $\theta^{3}$ indicates its position following the thickness direction, where $\theta^{3} \in[-H / 2, H / 2]$ ( $H$ is the local shell thickness). Partial derivatives of $\mathbf{R}\left(\theta^{\alpha}\right)$ define the covariant base vectors of the mid-surface :

$$
\mathbf{a}_{\alpha}=\frac{\partial \mathbf{R}\left(\theta^{\alpha}\right)}{\partial \theta^{\alpha}}=\mathbf{R}, \alpha
$$

and $\mathbf{a}_{3}$ represents the director vector of the shell structure. Hence, at an arbitrary material point belonging to the shell continuum, the covariant base vectors at the reference configuration are given by

$$
\begin{aligned}
& \mathbf{G}_{\alpha}=\mathbf{X},_{\alpha}=\mathbf{a}_{\alpha}+\theta^{3} \mathbf{a}_{3, \alpha}, \\
& \mathbf{G}_{3}=\mathbf{X},_{3}=\mathbf{a}_{3} .
\end{aligned}
$$

In a similar manner as compared with Equation (1), the position vector $\mathbf{x}$ of a material point in the current configuration can be defined by the following expression:

$$
\mathbf{x}\left(\theta^{\alpha}, \theta^{3}\right)=\mathbf{r}\left(\theta^{\alpha}\right)+\theta^{3} \overline{\mathbf{a}}_{3}\left(\theta^{\alpha}\right),
$$

where $\mathbf{r}$ and $\overline{\mathbf{a}}_{3}$ refer, respectively, to the updated vectors of the previously defined $\mathbf{R}$ and $\mathbf{a}_{3}$ in the current configuration. Similarly to Equation (2), we define the covariant base at the mid-surface for the current configuration:

$$
\overline{\mathbf{a}}_{\alpha}=\frac{\partial \mathbf{r}\left(\theta^{\alpha}\right)}{\partial \theta^{\alpha}}=\mathbf{r}, \alpha
$$

Transporting $\mathbf{X}$ to $\mathbf{x}$ is based on definition of a translational vector $\mathbf{v}$, acting on $\mathbf{R}$ at the mid-surface, and of a difference vector $\mathbf{w}$ updating the director $\mathbf{a}_{3}$ :

$$
\mathbf{r}=\mathbf{R}+\mathbf{v} \quad, \quad \overline{\mathbf{a}}_{3}=\mathbf{a}_{3}+\mathbf{w},
$$

where $\overline{\mathbf{a}}_{3}$ is the director vector in the current configuration. Equation (6) together with position vectors Equations (1) and (4) accounting, respectively, for the reference and current configuration allow us to define the displacement vector $\mathbf{u}$, which is associated with an arbitrary material point of the shell body:

$$
\mathbf{u}\left(\theta^{\alpha}, \theta^{3}\right)=\mathbf{x}\left(\theta^{\alpha}, \theta^{3}\right)-\mathbf{X}\left(\theta^{\alpha}, \theta^{3}\right)=\mathbf{v}\left(\theta^{\alpha}\right)+\theta^{3} \mathbf{w}\left(\theta^{\alpha}\right) .
$$

In total, six degrees of freedom can be distinguished in Equation (7) to describe the shell kinematics: we count three vector components $v_{1}, v_{2}, v_{3}$ relative to translation of the mid-surface and other three components $w_{1}, w_{2}, w_{3}$ updating the director vector. 


\subsection{Formulation for linear seven-parameter shell}

We derive in the following the shell displacement field within linear context in order to develop the subsequent multiscale framework based on microscopic small strains. Hence,

$$
\varepsilon_{i j}^{u}=\frac{1}{2}\left(\mathbf{G}_{i} \cdot \frac{\partial \mathbf{u}}{\partial \theta^{j}}+\mathbf{G}_{j} \cdot \frac{\partial \mathbf{u}}{\partial \theta^{i}}\right) .
$$

The superscript $(\cdot)^{u}$ refers to compatible quantities derived from displacement. Then,

$$
\begin{aligned}
\varepsilon_{\alpha \beta}^{u} & =\frac{1}{2}\left[\left(\mathbf{a}_{\alpha}+\theta^{3} \mathbf{a}_{3, \alpha}\right) \cdot\left(\mathbf{v}_{, \beta}+\theta^{3} \mathbf{w}_{, \beta}\right)+\left(\mathbf{a}_{\beta}+\theta^{3} \mathbf{a}_{3, \beta}\right) \cdot\left(\mathbf{v}_{, \alpha}+\theta^{3} \mathbf{w}_{, \alpha}\right)\right], \\
\varepsilon_{\alpha 3}^{u} & =\frac{1}{2}\left[\left(\mathbf{a}_{\alpha}+\theta^{3} \mathbf{a}_{3, \alpha}\right) \cdot \mathbf{w}+\mathbf{a}_{3} \cdot\left(\mathbf{v}_{, \alpha}+\theta^{3} \mathbf{w}_{, \alpha}\right)\right] \\
\varepsilon_{33}^{u} & =\mathbf{a}_{3} \cdot \mathbf{w} .
\end{aligned}
$$

Indices $(\alpha, \beta) \in(1,2)$. Expanding the strain tensor expression (9) gives terms of order 0, which are independent of $\theta^{3}$, and terms of order 1 , which are linear with respect to $\theta^{3}$. By neglecting the terms associated with $\left(\theta^{3}\right)^{2}$, we obtain

$$
\varepsilon_{i j}^{u}=\varepsilon_{i j}^{u(0)}+\theta^{3} \varepsilon_{i j}^{u(1)} .
$$

In order to better understand the kinematic significance of each strain tensor component, we develop in the following the detailed expressions of the components in (10). For reasons of clear illustration, the reference configuration is set in a fixed Cartesian system. This assumption is made only for demonstrative reasons and does not affect the generality of the proposed method. We adopt the following notations:

$$
\mathbf{a}_{i} \cdot \mathbf{v}=v_{i} \quad, \quad \mathbf{a}_{i} \cdot \mathbf{w}=w_{i} \quad, \quad \mathbf{a}_{3, \alpha}=0 .
$$

Then, components of $\varepsilon_{i j}^{u(0)}$ are expressed as follows:

$$
\begin{aligned}
\varepsilon_{11}^{u(0)} & =v_{1,1}, \\
\varepsilon_{12}^{u(0)} & =\frac{1}{2}\left(v_{1,2}+v_{2,1}\right)=\varepsilon_{21}^{u(0)}, \\
\varepsilon_{13}^{u(0)} & =\frac{1}{2}\left(w_{1}+v_{3,1}\right)=\varepsilon_{31}^{u(0)}, \\
\varepsilon_{22}^{u(0)} & =v_{2,2}, \\
\varepsilon_{23}^{u(0)} & =\frac{1}{2}\left(w_{2}+v_{3,2}\right)=\varepsilon_{23}^{u(0)}, \\
\varepsilon_{33}^{u(0)} & =w_{3} .
\end{aligned}
$$

Similarly, the strain tensor $\varepsilon_{i j}^{u(1)}$ can be written as

$$
\begin{aligned}
\varepsilon_{11}^{u(1)} & =w_{1,1}, \\
\varepsilon_{12}^{u(1)} & =\frac{1}{2}\left(w_{1,2}+w_{2,1}\right)=\varepsilon_{21}^{u(1)}, \\
\varepsilon_{13}^{u(1)} & =\frac{1}{2} w_{3,1}=\varepsilon_{31}^{u(1)}, \\
\varepsilon_{22}^{u(1)} & =w_{2,2}, \\
\varepsilon_{23}^{u(1)} & =\frac{1}{2} w_{3,2}=\varepsilon_{32}^{u(1)}, \\
\varepsilon_{33}^{u(1)} & =0 .
\end{aligned}
$$


We then obtain by integration the generalized stress resultants, among which the membrane forces, denoted by $\mathbf{n}$ :

$$
\mathbf{n}=\int_{H} \sigma\left(\theta^{3}\right) \mathrm{d} \theta^{3},
$$

and the generalized moment, denoted by $\mathbf{m}$ :

$$
\mathbf{m}=\int_{H} \theta^{3} \sigma\left(\theta^{3}\right) \mathrm{d} \theta^{3},
$$

where

$$
\sigma(\mathbf{x})=\mathbb{C}(\mathbf{x}): \varepsilon(\mathbf{x})
$$

is the Cauchy stress and $\mathbb{C}$ is the fourth-order elastic modulus of the medium. In both expressions, integration is performed along the shell thickness $H$.

\section{SHELL HOMOGENIZATION FORMULATION WITHIN LINEAR FRAMEWORK}

\subsection{Localization problem and definition of effective quantities}

We assume that the material is heterogeneous and can be characterized by a periodic microstructure, which by duplicating itself along the tangential directions of the shell, constitutes the macroscale heterogeneous shell, as depicted in Figure 2. The microstructure occupies a domain $\omega$ in $\mathbb{R}^{3}$, with its external boundary noted by $\partial \omega$. The open domain associated with $\omega$ is denoted by $\bar{\omega}$. In what follows, we assume that the elementary cell (RVE) is defined in Cartesian coordinates $\left(x_{1}, x_{2}, x_{3}\right)$ associated with the local curvilinear coordinate system.

We first define the strain and stress in-plane averaged quantities:

$$
\hat{\boldsymbol{\varepsilon}}\left(x_{3}\right)=\left\langle\boldsymbol{\varepsilon}\left(x_{1}, x_{2}, x_{3}\right)\right\rangle_{S}\left(x_{3}\right)=\frac{1}{S} \int_{S} \boldsymbol{\varepsilon}(\mathbf{x}) d S,
$$

where $S$ refers to the in-plane section area (as indicated in Figure 2) of the elementary cell, $d S=$ $d x_{1} d x_{2}$ in a Cartesian frame, and

$$
\hat{\boldsymbol{\sigma}}\left(x_{3}\right)=\left\langle\boldsymbol{\sigma}\left(x_{1}, x_{2}, x_{3}\right)\right\rangle_{S}\left(x_{3}\right)=\frac{1}{S} \int_{S} \boldsymbol{\sigma}(\mathbf{x}) d S .
$$

In the following, the macroscopic strain field is assumed to be identified with (10):

$$
\langle\boldsymbol{\varepsilon}(\mathbf{x})\rangle_{S}\left(x_{3}\right)=\overline{\hat{\varepsilon}}\left(x_{3}\right)=\overline{\boldsymbol{\varepsilon}}^{0}+x_{3} \overline{\boldsymbol{\varepsilon}}^{1} .
$$

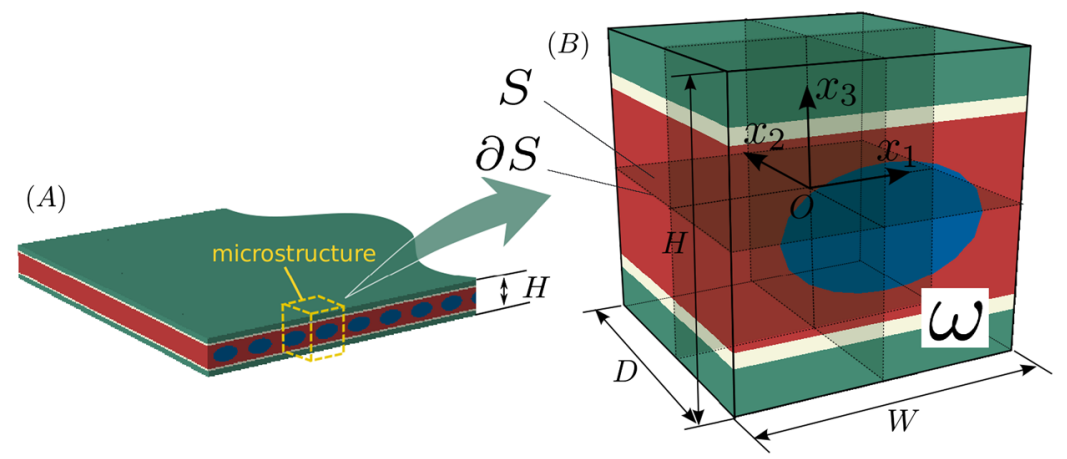

Figure 2. (A) Fully detailed thin structure; (B) periodic unit cell associated with the microstructure. 
In the preceding equation, $\overline{\boldsymbol{\varepsilon}}^{0}$ and $\overline{\boldsymbol{\varepsilon}}^{1}$ are the zeroth-order and first-order macroscopic strain contributions, identified as $\boldsymbol{\varepsilon}^{u,(0)}$ and $\boldsymbol{\varepsilon}^{u,(1)}$, respectively, in (10).

The localization problem is then to find $\boldsymbol{\varepsilon}(\mathbf{x})$, given $\overline{\boldsymbol{\varepsilon}}^{0}$ and $\overline{\boldsymbol{\varepsilon}}^{1}$ and verifying

$$
\nabla \cdot \sigma(\mathbf{x})=0 \text { in } \bar{\omega},
$$

and where the strain tensor is assumed to satisfy (19). Equation (19) can be verified for appropriate boundary conditions on the RVE, which will be specified in Section 3.2.

The weak form associated to Equation (20) is to find $\mathbf{u}(\mathbf{x})$ satisfying Dirichlet boundary conditions and $\mathbf{u}(\mathbf{x}) \in H^{1}(\omega)$ such that

$$
\int_{\omega} \boldsymbol{\sigma}(\mathbf{x}): \delta \boldsymbol{\varepsilon}(\mathbf{x}) d \Omega=0
$$

for all $\delta \mathbf{u} \in H_{0}^{1}(\omega)$, where $\delta \boldsymbol{\varepsilon}(\mathbf{x})=\varepsilon(\delta \mathbf{u}(\mathbf{x}))$.

\subsection{Microscopic boundary conditions}

The macroscopic strain field can then be decomposed according to

$$
\boldsymbol{\varepsilon}\left(x_{1}, x_{2}, x_{3}\right)=\overline{\hat{\boldsymbol{\varepsilon}}}\left(x_{3}\right)+\tilde{\boldsymbol{\varepsilon}}\left(x_{1}, x_{2}, x_{3}\right),
$$

where $\tilde{\boldsymbol{\varepsilon}}\left(x_{1}, x_{2}, x_{3}\right)$ is a perturbation due to the presence of heterogeneities in both $x_{1}, x_{2}$, and $x_{3}$ directions. Condition (19) can be verified by prescribing boundary conditions as follows. Considering (22), integration over the surface $S$ for a fixed value of $x_{3}$ gives

$$
\begin{aligned}
\left\langle\boldsymbol{\varepsilon}\left(x_{1}, x_{2}, x_{3}\right)\right\rangle_{S}\left(x_{3}\right) & =\overline{\hat{\boldsymbol{\varepsilon}}}\left(x_{3}\right)+\frac{1}{S} \int_{S} \tilde{\varepsilon}\left(x_{1}, x_{2}, x_{3}\right) d S, \\
& =\overline{\hat{\boldsymbol{\varepsilon}}}\left(x_{3}\right)+\frac{1}{2 S} \int_{\partial S} \tilde{\mathbf{u}} \otimes \mathbf{n}+\mathbf{n} \otimes \tilde{\mathbf{u}} d l,
\end{aligned}
$$

where $\partial S$ the boundary of the surface $S$ (Figure 2) for a laminate at $x_{3}$. Equation (23) is verified at $x_{3}$ if the surface integral vanishes, which holds if $\tilde{\mathbf{u}}$ is periodic in the $x_{1}$ and $x_{2}$ directions over $S$, or if $\tilde{\mathbf{u}}=0$ on $\partial S$, for a fixed value of $x_{3}$. In the present work, we consider the first condition. From (22), we obtain the following boundary conditions, which are applied on the lateral boundaries of the RVE (along $x_{1}$ and $x_{2}$ directions; Figure 2):

$$
\mathbf{u}(\mathbf{x})=\overline{\hat{\boldsymbol{\varepsilon}}}\left(x_{3}\right) \mathbf{x}+\tilde{\mathbf{u}}(\mathbf{x}) .
$$

Then, 12 elementary problems must be solved on the microscopic domain $\omega$, corresponding to the following macroscopic strains:

$$
\overline{\hat{\boldsymbol{\varepsilon}}}^{(k l), 0}\left(x_{3}\right)=\overline{\boldsymbol{\varepsilon}}^{(k l), 0}+x_{3} \overline{\boldsymbol{\varepsilon}}^{(k l), 1}
$$

with

$$
\begin{gathered}
\overline{\boldsymbol{\varepsilon}}^{(11), 0}=\left[\begin{array}{lll}
1 & 0 & 0 \\
0 & 0 & 0 \\
0 & 0 & 0
\end{array}\right] ; \overline{\boldsymbol{\varepsilon}}^{(22), 0}=\left[\begin{array}{lll}
0 & 0 & 0 \\
0 & 1 & 0 \\
0 & 0 & 0
\end{array}\right] ; \overline{\boldsymbol{\varepsilon}}^{(33), 0}=\left[\begin{array}{lll}
0 & 0 & 0 \\
0 & 0 & 0 \\
0 & 0 & 1
\end{array}\right] ; \\
\overline{\boldsymbol{\varepsilon}}^{(12), 0}=\left[\begin{array}{ccc}
0 & 1 / 2 & 0 \\
1 / 2 & 0 & 0 \\
0 & 0 & 0
\end{array}\right] ; \overline{\boldsymbol{\varepsilon}}^{(13), 0}=\left[\begin{array}{ccc}
0 & 0 & 1 / 2 \\
0 & 0 & 0 \\
1 / 2 & 0 & 0
\end{array}\right] ; \overline{\boldsymbol{\varepsilon}}^{(23), 0}=\left[\begin{array}{ccc}
0 & 0 & 0 \\
0 & 0 & 1 / 2 \\
0 & 1 / 2 & 0
\end{array}\right] ; \\
\overline{\boldsymbol{\varepsilon}}^{(k l), 1}=x_{3} \overline{\boldsymbol{\varepsilon}}^{(k l), 0}, \quad k=1, \ldots, 3, \quad l=1, \ldots, 3 .
\end{gathered}
$$




\subsection{Effective constitutive relationships and macroscopic problem}

Invoking the superposition principle, the microscopic strain field can be expressed in $\bar{\omega}$ as

$$
\boldsymbol{\varepsilon}(\mathbf{x})=\mathbb{A}^{0}(\mathbf{x}): \overline{\boldsymbol{\varepsilon}}^{0}+\mathbb{A}^{1}(\mathbf{x}): \overline{\boldsymbol{\varepsilon}}^{1},
$$

where $\mathbb{A}^{0}(\mathbf{x})$ and $\mathbb{A}^{1}(\mathbf{x})$ are fourth-order tensors such that

$$
A_{i j k l}^{0}(\mathbf{x})=\varepsilon_{i j}^{(k l), 0}(\mathbf{x}), \quad A_{i j k l}^{1}(\mathbf{x})=\varepsilon_{i j}^{(k l), 1}(\mathbf{x}),
$$

where $\varepsilon_{i j}^{(k l), 0}(\mathbf{x})$ is the strain field obtained by solving problems (20)-(19) with boundary conditions (24), for $\overline{\boldsymbol{\varepsilon}}^{(k l), 0}=\frac{1}{2}\left(\mathbf{e}_{k} \otimes \mathbf{e}_{l}+\mathbf{e}_{l} \otimes \mathbf{e}_{k}\right)$ and $\overline{\boldsymbol{\varepsilon}}^{1}=\mathbf{0}$ and. Similarly, $\varepsilon_{i j}^{(k l), 1}(\mathbf{x})$ is the strain field obtained by solving the problem (20)-(19) with boundary condition (24), with $\overline{\boldsymbol{\varepsilon}}^{0}=\mathbf{0}$ and $\overline{\boldsymbol{\varepsilon}}^{(k l), 1}=$ $\frac{1}{2}\left(\mathbf{e}_{k} \otimes \mathbf{e}_{l}+\mathbf{e}_{l} \otimes \mathbf{e}_{k}\right)$.

Then, using the constitutive law (16) and (29), we have

$$
\sigma(\mathbf{x})=\mathbb{C}(\mathbf{x}): \mathbb{A}^{0}(\mathbf{x}): \bar{\varepsilon}^{0}+\mathbb{C}(\mathbf{x}): \mathbb{A}^{1}(\mathbf{x}): \overline{\boldsymbol{\varepsilon}}^{1} .
$$

Using (14), (18), and (31), we obtain

$$
\overline{\mathbf{n}}=\frac{1}{S} \int_{H} \int_{S} \sigma(\mathbf{x}) d S d H=\frac{1}{S} \int_{\omega} \sigma(\mathbf{x}) d \Omega .
$$

Similarly, using (15), (18) and (31), we obtain:

$$
\overline{\mathbf{m}}=\frac{1}{S} \int_{H} \int_{S} x_{3}[\boldsymbol{\sigma}(\mathbf{x}) d S] d H=\frac{1}{S} \int_{\omega} x_{3} \sigma(\mathbf{x}) d \Omega .
$$

It yields, by using (32), (33), and (31), the following effective constitutive relationships:

$$
\begin{aligned}
& \overline{\mathbf{n}}=\overline{\mathbb{C}}^{11}: \overline{\boldsymbol{\varepsilon}}^{0}+\overline{\mathbb{C}}^{12}: \overline{\boldsymbol{\varepsilon}}^{1}, \\
& \overline{\mathbf{m}}=\overline{\mathbb{C}}^{21}: \overline{\boldsymbol{\varepsilon}}^{0}+\overline{\mathbb{C}}^{22}: \overline{\boldsymbol{\varepsilon}}^{1},
\end{aligned}
$$

with

$$
\begin{aligned}
\overline{\mathbb{C}}^{11}=\frac{1}{S} \int_{\omega} \mathbb{C}(\mathbf{x}): \mathbb{A}^{0}(\mathbf{x}) d \Omega, & \overline{\mathbb{C}}^{12}=\frac{1}{S} \int_{\omega} \mathbb{C}(\mathbf{x}): \mathbb{A}^{1}(\mathbf{x}) d \Omega, \\
\overline{\mathbb{C}}^{21}=\frac{1}{S} \int_{\omega} x_{3} \mathbb{C}(\mathbf{x}): \mathbb{A}^{0}(\mathbf{x}) d \Omega, & \overline{\mathbb{C}}^{22}=\frac{1}{S} \int_{\omega} x_{3} \mathbb{C}(\mathbf{x}): \mathbb{A}^{1}(\mathbf{x}) d \Omega .
\end{aligned}
$$

Let $\Omega$ be a domain associated with the shell model and $\bar{\Omega}$ the associated open domain, the equilibrium equations associated with the macroscopic problem are expressed by

$$
\begin{aligned}
& \nabla \cdot \overline{\mathbf{n}}=0 \text { in } \bar{\Omega}, \\
& \nabla \cdot \overline{\mathbf{m}}=0 \text { in } \bar{\Omega},
\end{aligned}
$$

and are supplemented by boundary conditions on the boundary $\partial \Omega$ of $\Omega$. The associated weak form is given as follows.

Find $\overline{\mathbf{u}}$ satisfying the boundary conditions associated with the macroscopic problem and $\overline{\mathbf{u}} \in$ $H^{1}(\bar{\Omega})$ such that

$$
\int_{\Omega} \overline{\mathbf{n}}: \delta \overline{\boldsymbol{\varepsilon}}^{0} d \Omega+\int_{\Omega} \overline{\mathbf{m}}: \delta \overline{\boldsymbol{\varepsilon}}^{1} d \Omega=\lambda \int_{\partial \Omega_{F}} \mathbf{F} \cdot \delta \overline{\mathbf{u}} d \Gamma
$$

where $\mathbf{F}$ denotes prescribed traction on the Neumann boundary of the shell domain and $\lambda$ is a scalar parameter describing the intensity of the load. By introducing Equations (34) and (35) into Equation (40), we obtain the final form of the macroscopic problem to solve 


$$
\int_{\Omega}\left(\bar{\varepsilon}^{0}: \overline{\mathbb{C}}^{11}: \delta \bar{\varepsilon}^{0}+\overline{\boldsymbol{\varepsilon}}^{1}: \overline{\mathbb{C}}^{12}: \delta \overline{\boldsymbol{\varepsilon}}^{0}+\overline{\boldsymbol{\varepsilon}}^{0}: \overline{\mathbb{C}}^{21}: \delta \overline{\boldsymbol{\varepsilon}}^{1}+\overline{\boldsymbol{\varepsilon}}^{1}: \overline{\mathbb{C}}^{22}: \delta \overline{\boldsymbol{\varepsilon}}^{1}\right) d \Gamma=\lambda \int_{\partial \Omega_{F}} \mathbf{F} \cdot \delta \overline{\mathbf{u}} d \Omega
$$

The problem (41) can be solved by applying the shell finite element discretization that is detailed in Appendices A and B.

\section{EXTENSION OF THE APPROACH TO MACROSCOPIC GEOMETRICAL NONLINEARITIES}

Even though the assumption of small perturbations can be useful in some applications, many practical situations involving thin structures require considering finite displacements and rotations. More specifically, these structures can be subjected to structural instabilities such as buckling, due to their high aspect ratio. However, when choosing multiscale modeling strategies, the potential of sequential schemes based on elastic material homogenization is usually underestimated because they are easily associated with application limitations within linear context. In fact, because of the intrinsic properties of thin structures, an important number of problems based on shell structures showing significant displacements and rotations do not necessarily lead to large, but infinitesimal local material deformations. Examples exposed in the numerical test section (Sections 5.2 and 5.3; particularly see Figures 16 and 17) will confirm this fact. Hence, the validity of effective material laws obtained using the present homogenization scheme can be conveniently extended to macroscopic problems subjected to geometrical nonlinearities. The aim of this section is to provide this extension, in order to account for problems involving large displacements and rotations at the macroscopic level. In the case of finite strains, the compatible Green-Lagrange strain tensor for the shell model is derived from the displacement field [24]:

$$
E_{i j}^{u}=\frac{1}{2}\left(\mathbf{G}_{i} \cdot \mathbf{u}_{, j}+\mathbf{G}_{j} \cdot \mathbf{u}_{, i}+\mathbf{u}_{, i} \cdot \mathbf{u}_{, j}\right),
$$

where the last term $\left(\mathbf{u}_{, i} \cdot \mathbf{u}_{, j}\right)$ accounts for the geometrical nonlinearity which was not taken into account in previous sections. Similarly to Equation (10), the compatible strain field $E_{i j}^{u}$ can be rearranged with respect to the order of $\theta^{3}$ into a part of order 0 and a part of order 1 . So we have

$$
\mathbf{E}^{u}=\mathbf{E}^{u(0)}+\theta^{3} \mathbf{E}^{u(1)}
$$

where $\mathbf{E}^{(0)}$ and $\mathbf{E}^{(1)}$ can be expressed depending on the displacement field and the covariant base vectors $\mathbf{G}_{i}$ (Equation (3)) as

$$
\begin{aligned}
& E_{\alpha \beta}^{u(0)}=\frac{1}{2}\left(\mathbf{a}_{\alpha} \mathbf{v}_{, \beta}+\mathbf{a}_{\beta} \mathbf{v}_{, \alpha}+\mathbf{v}_{, \alpha} \mathbf{v}_{, \beta}\right), \\
& E_{\alpha 3}^{u(0)}=\frac{1}{2}\left(\mathbf{a}_{\alpha} \mathbf{w}+\mathbf{a}_{3} \mathbf{v}_{, \alpha}+\mathbf{v}_{, \alpha} \mathbf{w}\right) \\
& E_{33}^{u(0)}=\mathbf{a}_{3} \mathbf{w}
\end{aligned}
$$

and for strains at order 1 ,

$$
\begin{aligned}
& E_{\alpha \beta}^{u(1)}=\frac{1}{2}\left(\mathbf{a}_{3, \alpha} \mathbf{v}_{, \beta}+\mathbf{a}_{\alpha} \mathbf{w}_{, \beta}+\mathbf{a}_{\beta} \mathbf{w}_{, \alpha}+\mathbf{a}_{3, \alpha} \mathbf{v}_{, \alpha}+\mathbf{v}_{, \alpha} \mathbf{w}_{, \beta}+\mathbf{w}_{, \alpha} \mathbf{v}_{, \beta}\right), \\
& E_{\alpha 3}^{u(1)}=\frac{1}{2}\left(\mathbf{a}_{3, \alpha} \mathbf{w}+\mathbf{a}_{3} \mathbf{w}_{, \alpha}+\mathbf{w}_{, \alpha} \mathbf{w}\right) \\
& E_{33}^{u(1)}=0 .
\end{aligned}
$$

Here, $E_{33}^{u(1)}=0$ but is enhanced by the additional assumed strain term $\tilde{E}_{33}$, which is introduced by using the condition of orthogonality (Equation (A.6)) with respect to the stress field. Readers are invited to refer to Appendix A for details. 
In the context of finite strains, the localization problem is nonlinear, and the superposition principle does not hold. We then propose an empirical effective model for the constitutive law as follows, which relates linearly the generalized stress resultants with respect to the macroscopic Green-Lagrange strains $\mathbf{E}^{(0)}$ and $\mathbf{E}^{(1)}$ :

$$
\begin{aligned}
& \overline{\mathbf{N}}=\overline{\mathbb{C}}^{11}: \overline{\mathbf{E}}^{0}+\overline{\mathbb{C}}^{12}: \overline{\mathbf{E}}^{1}, \\
& \overline{\mathbf{M}}=\overline{\mathbb{C}}^{21}: \overline{\mathbf{E}}^{0}+\overline{\mathbb{C}}^{22}: \overline{\mathbf{E}}^{1},
\end{aligned}
$$

in which $\overline{\mathbb{C}}^{11}, \overline{\mathbb{C}}^{12}, \overline{\mathbb{C}}^{21}$, and $\overline{\mathbb{C}}^{22}$ are given by the homogenization procedures prescribed on the unit cell by considering small strains (Equations (36) and (37)). The aforementioned model does not result from a micromechanical analysis, but we can, however, note that when local strains are small, the aforementioned model rigorously describes the effective model related to the homogenized shell presented in small strain context. Then, the macroscopic equilibrium equations are given by

$$
\nabla_{X} \cdot \overline{\mathbf{N}}=0
$$

and

$$
\nabla_{X} \cdot \overline{\mathbf{M}}=0
$$

where $\nabla_{X}($.$) denotes the divergence operator with respect to the reference configuration. The$ associated weak form writes

$$
\int_{\Omega} \overline{\mathbf{N}}: \delta \overline{\mathbf{E}}^{0} d \Omega+\int_{\Omega} \overline{\mathbf{M}}: \delta \overline{\mathbf{E}}^{1} d \Omega=\lambda \int_{\partial \Omega_{F}} \mathbf{F} \cdot \delta \overline{\mathbf{u}} d \Gamma,
$$

which gives by taking into account Equation (46)

$$
\int_{\Omega} \overline{\mathbf{E}}^{(0)}: \overline{\mathbb{C}}^{11}: \delta \overline{\mathbf{E}}^{(0)}+\overline{\mathbf{E}}^{(1)}: \overline{\mathbb{C}}^{12}: \delta \overline{\mathbf{E}}^{(0)}+\overline{\mathbf{E}}^{(0)}: \overline{\mathbb{C}}^{21}: \delta \overline{\mathbf{E}}^{(1)}+\overline{\mathbf{E}}^{(1)}: \overline{\mathbb{C}}^{22}: \delta \overline{\mathbf{E}}^{(1)} d \Omega=\lambda \int_{\partial \Omega_{F}} \mathbf{F} \cdot \delta \overline{\mathbf{u}} d \Gamma .
$$

\section{NUMERICAL EXAMPLES}

The purpose of this section is to evaluate the capability of the proposed multiscale technique to model the response of heterogeneous structures, consisting here as multilayer composite materials with or without fiber reinforcement. Different loading conditions are designed so as to investigate various kinematics ranging from simple bending to large displacements and rotations. In all cases, large displacements are taken into account, and the effective moduli of the homogenized shell model have been obtained by the procedure described in Section 3 and used within the nonlinear framework of Section 4. The reference solutions involving the fully meshed model or other reference models have been obtained through ABAQUS [25] software (Abaqus Inc., Vélizy-Villacoublay, France).

\subsection{Bending of a composite thin plate}

The first numerical example deals with the bending of a heterogeneous thin plate. As depicted in Figure 3, a five-layer composite rectangular thin plate with length $L_{M}=30$, width $l_{M}=10$, and thickness $H_{M}=1 \mathrm{~mm}$ is considered. Reinforce fibers with elliptical section are horizontally disposed in the central layer. Clamped on the left edge, the structure is submitted to the load force $F$, which is uniformly distributed on its right edge.

The microstructure is chosen so as to completely capture the heterogeneities across the shell thickness. The microstructure dimensions are $L_{m}=l_{m}=H_{m}=1 \mathrm{~mm}$, for which $H_{m}=H_{M}$ (Figure 4). In this example, the microstructure is composed of elastic linear materials. Material properties for each phase are given in Figure 4.

The six problems at order 0 and at order 1 are solved numerically on the elementary cell by means of the boundary conditions (24)-(27). The corresponding deformed configurations are depicted in 


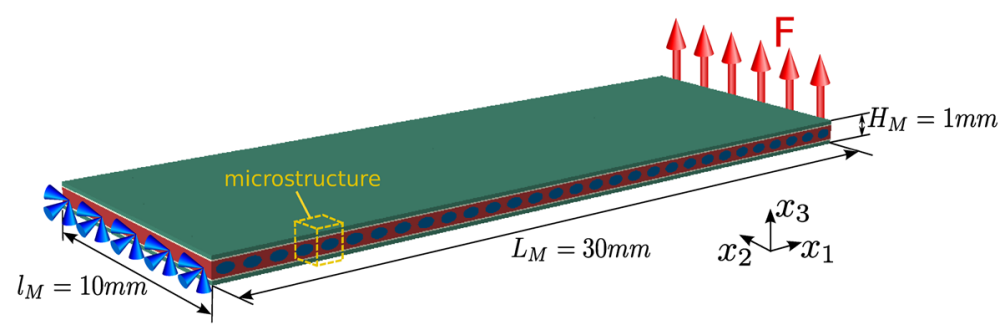

Figure 3. Geometry of the heterogeneous plate problem in bending.

\begin{tabular}{cc}
\hline$E(G P a)$ & $\nu$ \\
\hline 117 & 0.33 \\
68.9 & 0.33 \\
210 & 0.30 \\
379.2 & 0.30 \\
\hline
\end{tabular}

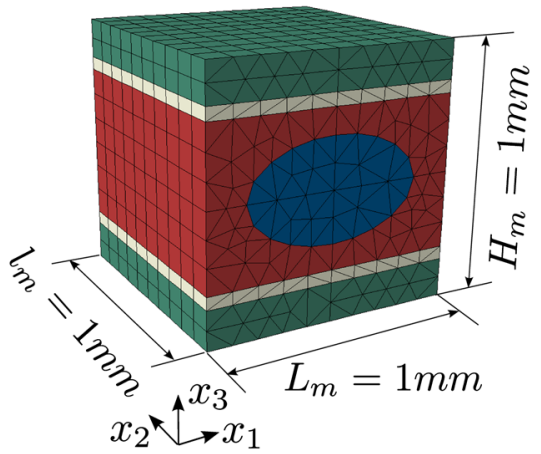

Figure 4. Unit cell associated with the heterogeneous plate: geometry and material parameters for the different phases.

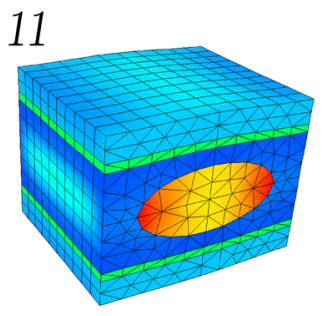

12

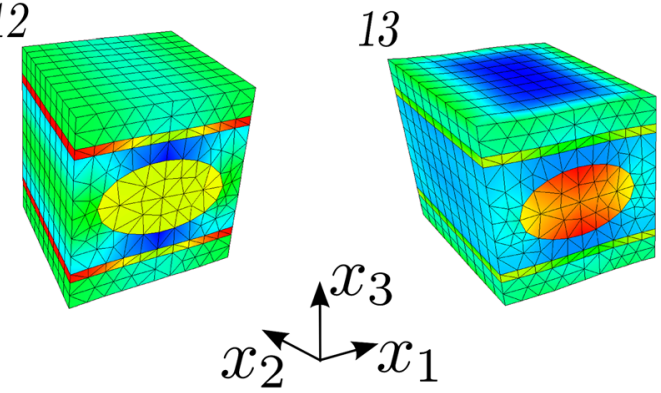

33

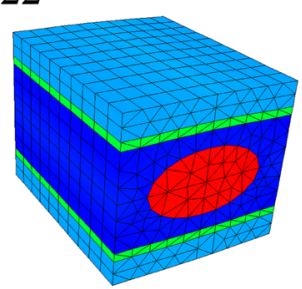

3

23
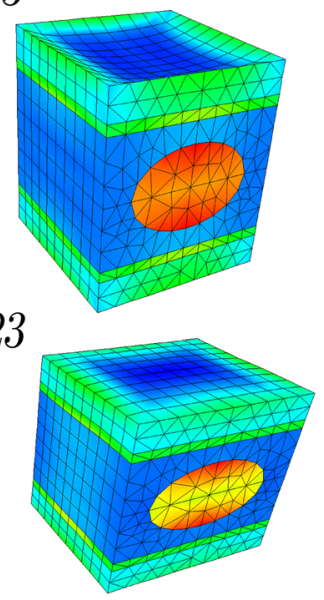

Figure 5. Deformed configurations associated with order 0 kinematic modes.

Figures 5 and 6 for illustration. Then, the effective constitutive tensors are computed according to the procedure described in Section 3.3.

In order to investigate the validity of the multiscale approach, the entire heterogeneous thin plate is fully modeled so as to provide a reference solution. In this example, the full-scale mesh contains 347,000 degrees of freedom and is discretized with the six-node wedge solid element. As for the macroscale boundary conditions, the full model and the homogenized 3D shell are clamped on one edge, which requires suppressing all related DOF on this edge including displacements and rotations. Then on the free edge, a force $F$ in $x_{3}$ direction is uniformly prescribed. The homogenized 


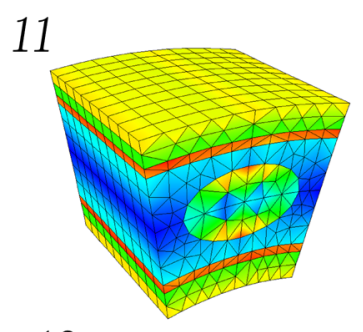

12

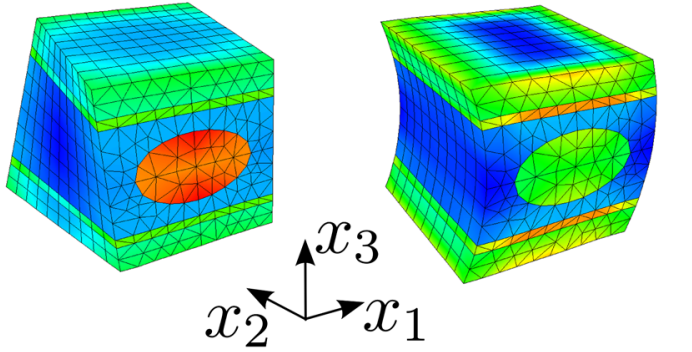

22

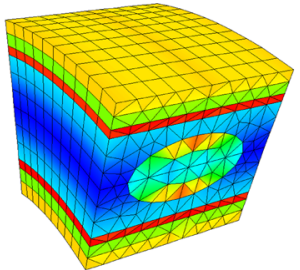

13
33

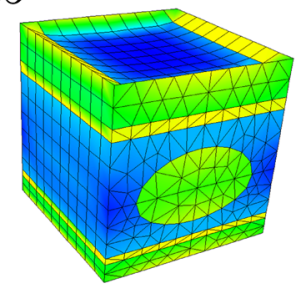

23

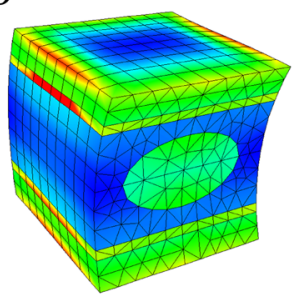

Figure 6. Deformed configurations associated with order 1 kinematic modes.

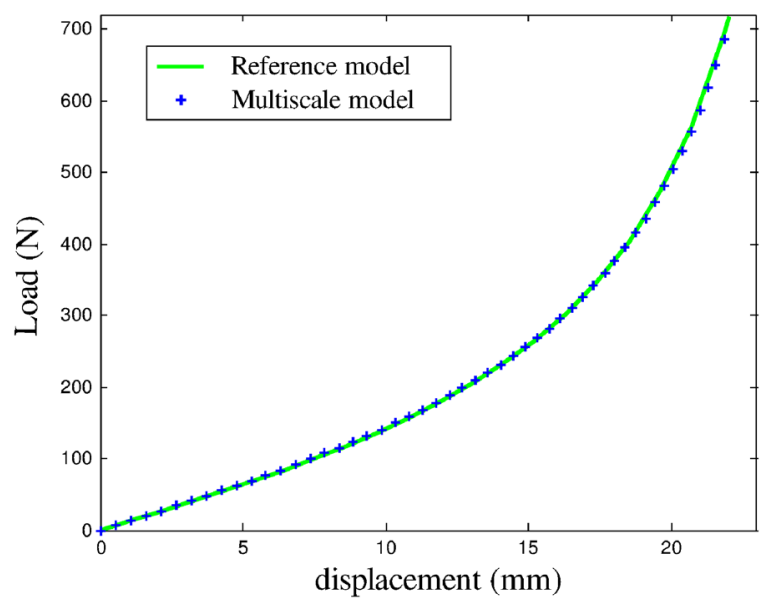

Figure 7. Load-displacement diagram for the heterogeneous plate bending problem measured at the loaded edge: comparison between the full-scale model and the homogenized shell model.

model is discretized with only 12 shell elements containing 318 DOF and is solved using NewtonRaphson resolution strategy with arc-length control (Riks' method [26, 27]), as well as for examples 5.2 and 5.3.

For both models on the right edge, we measure the displacement evolution in $x_{3}$ direction versus the load force. Results from the two models are compared in Figure 7. We note a very good agreement between the two results.

\subsection{Lateral buckling of a heterogeneous slender beam}

In this example, we consider a slender multilayer rectangular sheet, with length $L_{M}=100$, width $l_{M}=10$, and thickness $H_{M}=1 \mathrm{~mm}$. The sample is submitted to a concentrated load at the point $\mathrm{C}$ and is clamped at its left edge. Loading conditions are depicted in Figure 8. As the load increases, instabilities may occur with occurrence of a tiny perturbation. This perturbation is introduced to the system via a small force $f$, which is applied in the transverse direction. In this example, its intensity remains $1 / 1000$ of the load force $\lambda F$. 


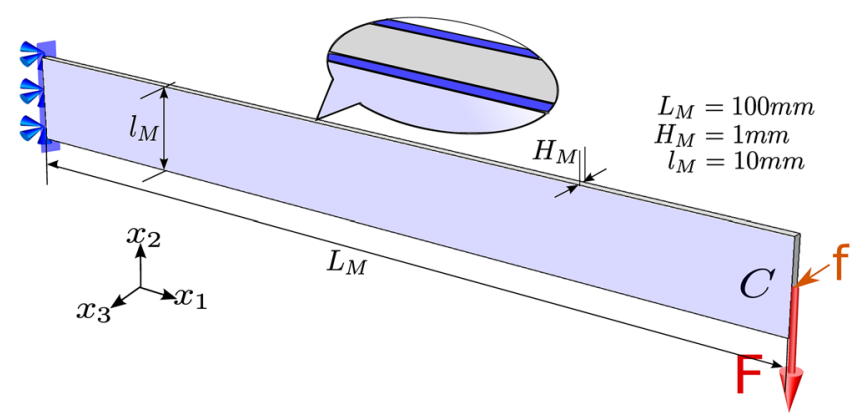

Figure 8. Thin heterogeneous plate under deflection: geometry and boundary conditions.

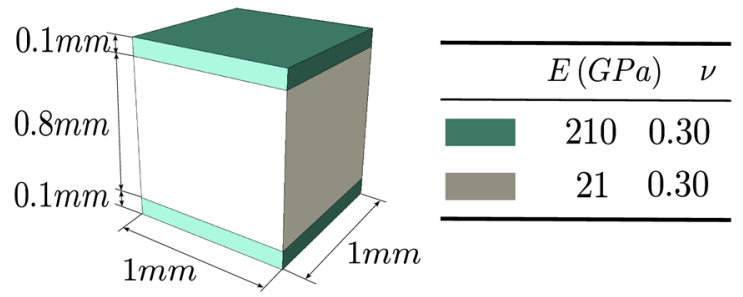

Figure 9. Unit cell associated with the heterogeneous plate under deflection: geometry and material parameters.

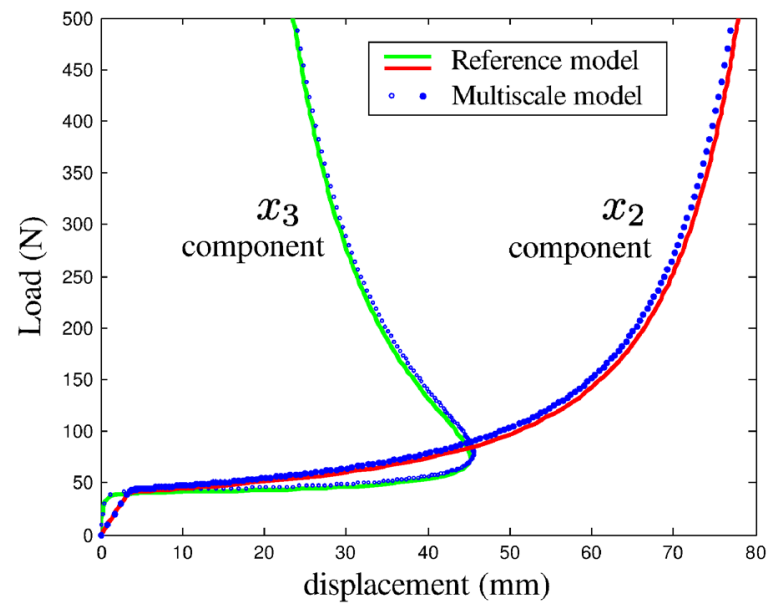

Figure 10. Load-displacement diagram for the heterogeneous plate bending problem: comparison between the full-scale model and the homogenized shell model: $x_{2}$ and $x_{3}$ components of the displacements at point C.

The considered thin plate represents a three-layer microstructure in the transverse direction. Material properties for each phase are considered linear elastic. Similar to the previous example, we model the microstructure such that the thin plate's structural details along the thickness direction are fully accounted for, as depicted in Figure 9.

The homogenized model (discretized with 12 elements including 318 DOF) is validated by comparing with a reference solution obtained with Abaqus. However, correctly modeling the present problem using full-scale solid elements, as it has been done in the first example, represents huge numerical difficulty for this case, due to the severe instabilities that the structure would suffer under the defined loading conditions. An alternative solution consists in using the multilayer shell elements that Abaqus provides, by explicitly defining the material properties for each shell layer.

We report in Figure 10 the results for the homogenized model in comparison with the Abaqus reference model. Displacement response in both the vertical direction $-x_{2}$ and the transverse direction 


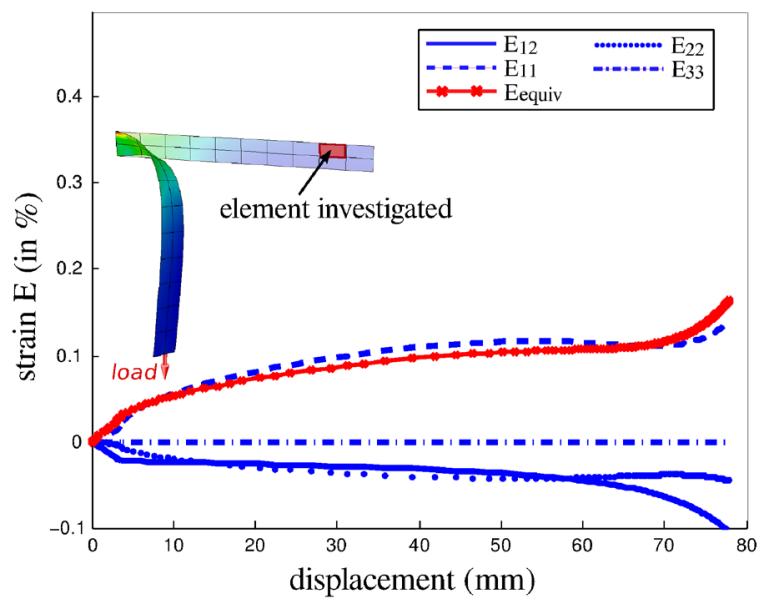

Figure 11. Element strain evolution of the macroscopic model. Strain values are set in local orthonormal material coordinates and expressed in per cent. The red curve refers to the equivalent strain.

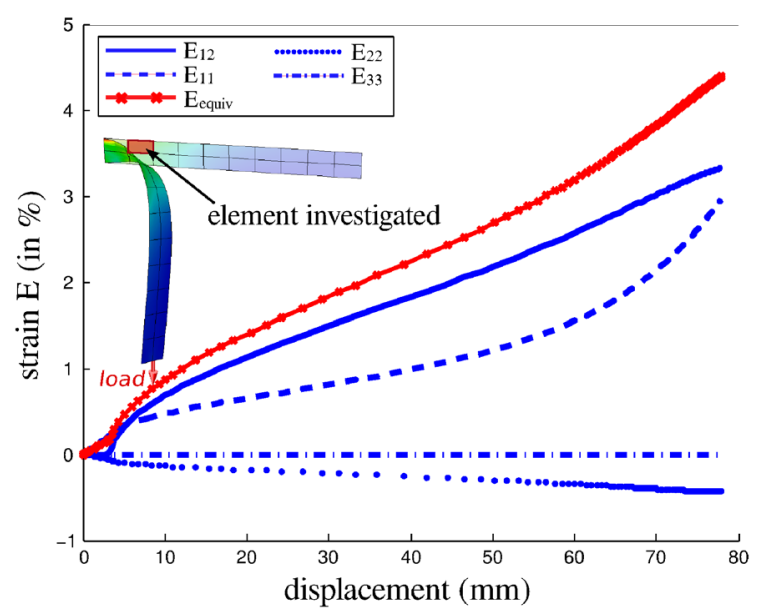

Figure 12. Element strain evolution of the macroscopic model. Strain values are set in local orthonormal material coordinates and expressed in per cent. The red curve refers to the equivalent strain.

$x_{3}$ are investigated as function of the increasing load. This example demonstrates the capability of the present multiscale framework in treating thin structures involving severe instabilities, especially when full-scale modeling using solid elements becomes difficult.

As to the question why the proposed sequential multiscale framework, based on elastic material homogenization can deal with severe macroscopic instabilities, we note that throughout the loading process, strains computed on the equivalent macroscopic model remain globally small (Figure 11). In severely deformed areas, a few elements present strain values close to $4 \%$ (Figure 12). However, these elements are situated near the boundary of the model, where modeling through multiscale methods is presumably difficult by nature. In fact, the most reliable way to model the boundary area would be using a full scale model which presents the most precise and the richest information. These models can then be coupled to the multiscale model by having recourse to model coupling techniques, for instance the Arlequin method [28]. Nevertheless, the multiscale model in the present case offered satisfactory accuracy.

\subsection{Snap-through buckling of a heterogeneous thin roof}

To fully illustrate the present procedure in the context of curved thin structures, we consider in this example the buckling behavior of a slightly curved thin roof composed of a five-layer material. 


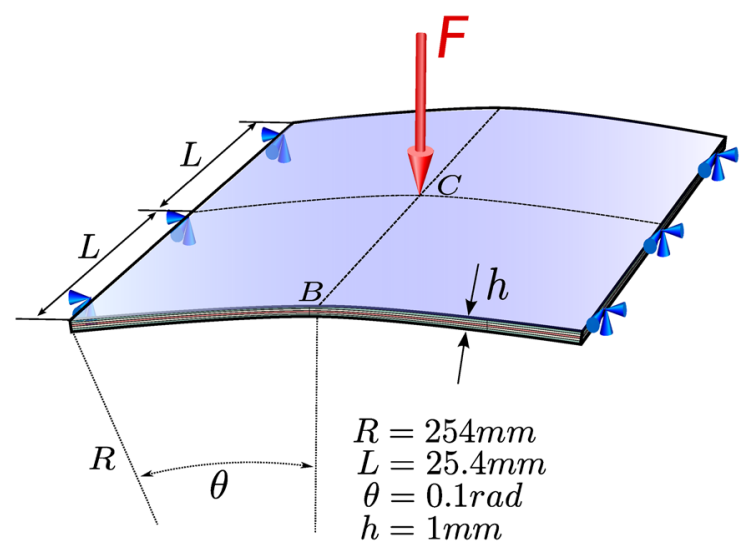

Figure 13. Curved thin heterogeneous roof problem: geometry and boundary conditions.

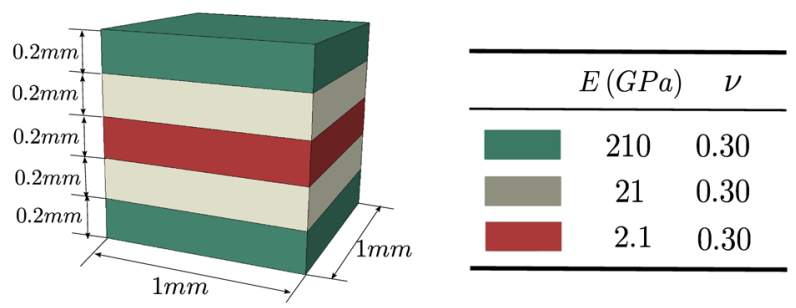

Figure 14. Unit cell associated with the curved thin heterogeneous roof : geometry and material parameters.

The geometry of the structure is shown in Figure 13. Both the left and right edges are clamped. A concentrated force is applied at point $\mathrm{C}$ situated at the center of the roof. Because of the curved geometry, the roof buckles as the load increases.

Similar to the previous case, the same thickness as the roof is retained for the microstructure which is modeled taking into account the five-layer microstructure. Material for each layer is linear elastic. The parameters of the different layers are provided in Figure 14.

For the sake of simplicity, only one quarter of the roof geometry is modeled because of the problem symmetry. Therefore, we used only nine shell elements (containing $240 \mathrm{DOF}$ ) to construct the homogenized model while the reference model is elaborated using Abaqus multilayer shell with the same number of elements. Displacement response in the transverse direction versus the loading force is computed both at the loading point $\mathrm{C}$ and on a point $\mathrm{B}$ situated on one of the roof's edges.

Results from the homogenized model are compared with those obtained from the reference model (Figure 15). Very good agreement can be noticed for displacements observed at both points B and C. This example further confirms the robustness of the proposed multiscale scheme in dealing with buckling problems for shell structures, which cannot be easily captured by full-scale solid element models, not to mention the computational cost it will induce.

Despite the large displacement involved in the macroscopic model, the macroscopic strains only evolve on a very low level. As depicted in Figures 16 and 17, strain values remain small throughout the loading process. This again explains why the proposed approach based on microscopic elastic homogenization is sufficient in spite of the complex macroscopic problem setting.

For the last two examples 5.2 and 5.3, we considered heterogeneous shells with simple multilayer microstructures. This choice has been made to facilitate modeling of the reference problem: on one hand, it is difficult to capture buckling behaviors of thin structures based on full-scale models with solid element; and on the other, Abaqus offers advanced possibility for modeling multilayer shells. However, as demonstrated in example 5.1, the present methodology is by no means restricted to multilayer microstructures and can be used for arbitrary microstructural geometries. 


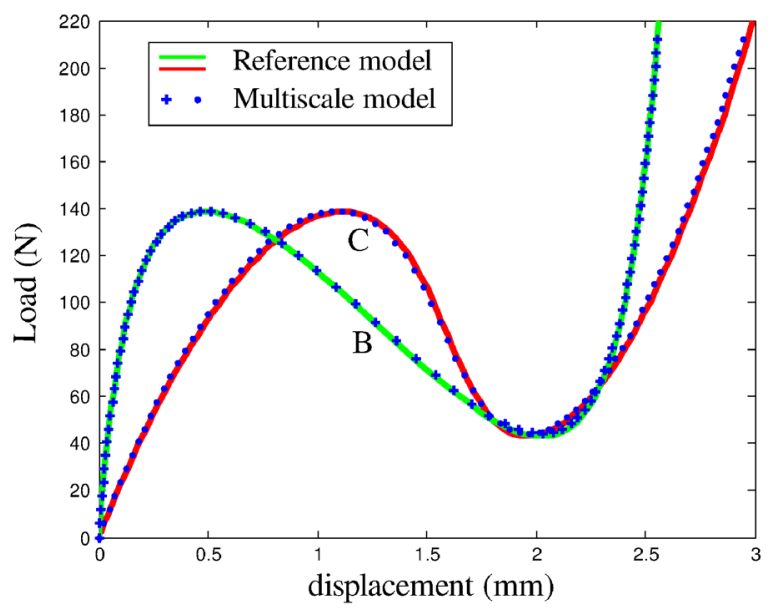

Figure 15. Load-displacement diagram for the roof buckling problem measured at point B and C: comparison between the reference model using Abaqus multilayer shell and the homogenized model.

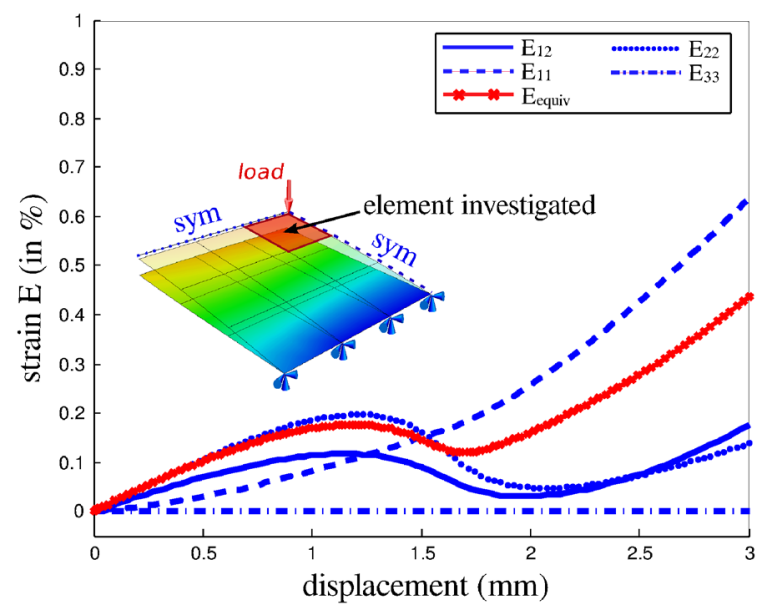

Figure 16. Element strain evolution of the macroscopic model. Strain values are set in local orthonormal material coordinates and expressed in per cent. The red curve refers to the equivalent strain.

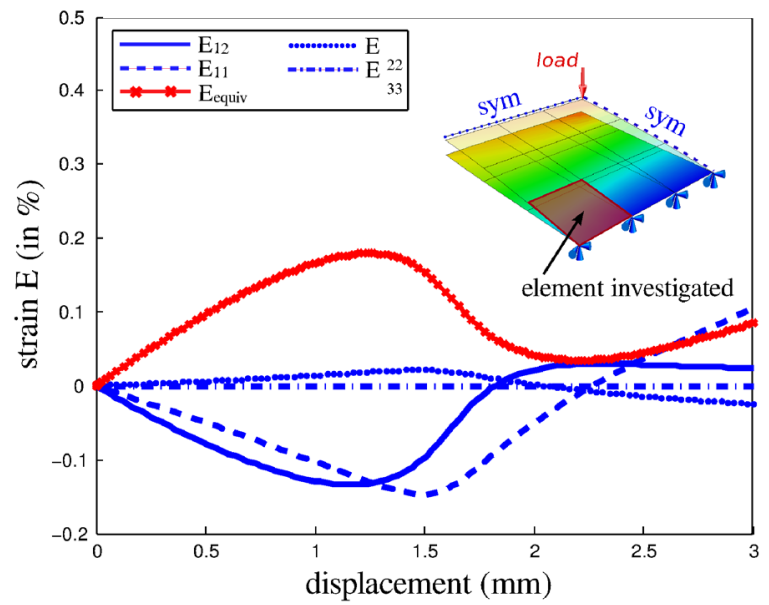

Figure 17. Element strain evolution of the macroscopic model. Strain values are set in local orthonormal material coordinates and expressed in per cent. The red curve refers to the equivalent strain. 


\section{CONCLUSION AND DISCUSSION}

In this work, a computational homogenization approach has been elaborated to deal with shell structures composed of multiphase elastic materials. Scale transition is achieved by solving a set of 12 boundary value problems prescribed on an elementary cell, where through thickness heterogeneities are modeled in details. At the macroscopic scale, a seven-parameter shell model is employed, with strain enrichment in the transverse direction. A numerical procedure has been defined to compute the different operators associated with the constitutive behavior at the macroscopic scale. The homogenization framework is first presented in the small strain context, and a simple extension to finite displacements and geometrical nonlinearities is then proposed, where a linear material behavior is chosen, based on the operators obtained from the small strain context but in a fully geometrical nonlinear context. The method has been validated through several examples involving heterogeneous plates and shells in large displacement with possible buckling, and very good agreement has been found between the homogenized model and reference solutions based on fully meshed structures where all heterogeneities are explicitly described. It should be noted, however, that validation of the presented approach has been carried out by comparing global variables such as displacements. If readers require accurately investigating local variables such as stresses, or studying microstructures composed of nonlinear materials under large deformation, a fully coupled concurrent type of homogenization scheme should be considered. Another possibility consists in prescribing full models in regions involving large strains and multiscale model in the remaining area, then coupling the two models using model coupling techniques, such as Arlequin method.

\section{APPENDIX A}

\section{A.1. Formulation of the seven-parameter shell element with enhanced assumed strain}

According to the shell kinematics assumptions provided in Section 2, deriving the displacement field leads to $E_{33}^{u(1)}=0$. However, this condition is not compatible with respect to three-dimensional continuum constitutive relations. In fact, for problems involving significant out-of-plane deformations, in-plane stretches and compressions such as $E_{11}^{u(1)}, E_{22}^{u(1)}$ induce by the Poisson effect a spurious strain term which is distributed linearly in the shell thickness direction:

$$
E_{33}^{\text {error }}=\frac{C_{33 i j}}{C_{3333}} \theta^{3} E_{i j}^{u(1)} \text { with }(i, j) \neq(3,3) .
$$

This spurious strain $E_{33}^{\text {error }}$ leads to significant error when it comes to bending dominated cases, which is commonly referred to as 'the Poisson thickness locking phenomenon'. To remedy this problem, we adopted in this work a versatile seven-parameter shell element incorporating enhanced assumed strain [29]. Proposed by Büchter and Ramm [22], the element is based on a multi-field formulation, for which the compatible strain field $\mathbf{E}^{u}$ is supplemented with a linear strain component $\tilde{\mathbf{E}}$ acting across the shell thickness direction. The additional strain balances $E_{33}^{\text {error }}$ and subsequently clears the locking issue. Incorporation of the additional strain is realized based on the three-field variational functional $\mathrm{Hu}$-Washizu, which is function of the second Piola-Kirchhoff stress $\mathbf{S}$, the Green-Lagrange strain $\mathbf{E}$ and displacement $\mathbf{u}$. According to $\mathrm{Hu}$-Washizu, total energy density of a system is

$$
\Pi_{H W}(\mathbf{u}, \mathbf{E}, \mathbf{S})=\int_{\Omega} \frac{1}{2} \mathbf{E}: \mathbb{C}: \mathbf{E}-\mathbf{S}:\left(\mathbf{E}-\mathbf{E}^{\mathbf{u}}\right) \mathrm{d} \Omega-\lambda P(\mathbf{u}),
$$

in which $P$ indicates the energy density due to external load, which is calibrated by a load parameter $\lambda$. $P$ is defined by

$$
P(\mathbf{u})=\int_{\Omega} \mathbf{f} \cdot \mathbf{u} \mathrm{d} \Omega+\int_{\partial \Omega} \mathbf{T} \cdot \mathbf{u} \mathrm{d} \Gamma,
$$


with $\mathbf{f}$ the volume force applied on the body $\Omega$ and $\mathbf{T}$ the surface force acting on the body boundary $\partial \Omega$. In Equation (A.2), $\mathbf{E}$ denotes the total strain, which is composed on one hand by the compatible strain $\mathbf{E}^{u}$ and on the other hand by the supplemented assumed strain $\tilde{\mathbf{E}}$ :

$$
\mathbf{E}=\mathbf{E}^{u}+\tilde{\mathbf{E}} .
$$

$\tilde{\mathbf{E}}$ describes linear variation of the shell thickness stretch (or compression) and is expressed with respect to the local transversal coordinates $\theta^{3}$ :

$$
\tilde{\mathbf{E}}=\theta^{3} \tilde{E}_{33} \mathbf{G}^{3} \otimes \mathbf{G}^{3} \quad \text { with } \tilde{E}_{33}=\tilde{E}_{33}\left(\theta^{1}, \theta^{2}\right) .
$$

Additionally, $\tilde{\mathbf{E}}$ satisfies the condition of orthogonality with respect to the stress field $\mathbf{S}$, which renders the following condition:

$$
\int_{\Omega} \mathbf{S}: \tilde{\mathbf{E}} \mathrm{d} \Omega=0
$$

Relations Equations (A.4) and (A.6) allows reformulating the $H u$-Washizu functional Equation (A.2):

$$
\Pi_{H W}(\mathbf{u}, \tilde{\mathbf{E}})=\int_{\Omega} \frac{1}{2} t\left(\mathbf{E}^{u}+\tilde{\mathbf{E}}\right): \mathbb{C}:\left(\mathbf{E}^{u}+\tilde{\mathbf{E}}\right) \mathrm{d} \Omega-\lambda P(\mathbf{u}) .
$$

From Equations (A.7) and (A.6), we obtain the variational definition of the problem, which is composed of an equilibrium equation and a compatibility condition:

$$
\left\{\begin{array}{l}
\int_{\Omega} \mathbf{S}: \delta \mathbf{E}^{u} \mathrm{~d} \Omega-\lambda P(\delta \mathbf{u})=0 \\
\int_{\Omega} \mathbf{S}: \delta \tilde{\mathbf{E}} \mathrm{d} \Omega=0,
\end{array}\right.
$$

where $P(\delta \mathbf{u})$ (coming from Equation (A.3)) represents energy density due to conservative external load, satisfying

$$
P(\delta \mathbf{u})=\int_{\Omega} \mathbf{f} \cdot \delta \mathbf{u} \mathrm{d} \Omega+\int_{\partial \Omega} \mathbf{T} \cdot \delta \mathbf{u} \mathrm{d} \Gamma .
$$

In Equation (A.8), because the compatible strain $\mathbf{E}^{u}$ derives from the displacement field $\mathbf{u}$

$$
\mathbf{E}^{u}=\frac{1}{2}\left(\nabla \mathbf{u}+\nabla^{T} \mathbf{u}\right)+\frac{1}{2} \nabla \mathbf{u} \cdot \nabla^{T} \mathbf{u},
$$

then its variational form written with respect to $\delta \mathbf{u}$ is

$$
\delta \mathbf{E}^{u}=\frac{1}{2}\left(\nabla \delta \mathbf{u}+\nabla^{T} \delta \mathbf{u}\right)+\nabla \mathbf{u} \cdot \nabla^{T} \delta \mathbf{u} .
$$

Let us adopt the operator $\mathbf{E}_{\mathbf{l}}^{\mathbf{u}}(\cdot)$ to denote $\frac{1}{2}\left[\nabla(\cdot)+{ }^{t} \nabla(\cdot)\right]$, and $\mathbf{E}_{n l}^{u}(*, \star)$ to denote $\frac{1}{2} \nabla(*)$. ${ }^{t} \nabla(\star)$. Then the variational definition of the problem writes

$$
\left\{\begin{array}{l}
\int_{\Omega} \mathbf{S}:\left[\mathbf{E}_{l}^{\mathbf{u}}(\delta \mathbf{u})+2 \mathbf{E}_{n l}^{u}(\mathbf{u}, \delta \mathbf{u})\right] \mathrm{d} \Omega=\lambda P(\delta \mathbf{u}) \\
\int_{\Omega} \mathbf{S}: \delta \tilde{\mathbf{E}} \mathrm{d} \Omega=0 \\
\mathbf{S}=\mathbb{C}:\left[\mathbf{E}_{\mathbf{l}}^{\mathbf{u}}(\mathbf{u})+\mathbf{E}_{n l}^{u}(\mathbf{u}, \mathbf{u})+\tilde{\mathbf{E}}\right],
\end{array}\right.
$$

which can be readily implemented using the discretization technique that we present in the next section. 


\section{A.2. Finite element discretization}

We introduce the discretization technique for the seven-parameter shell formulation with enhanced assumed strain. An eight-node quadrilateral element with reduced integration is utilized for which nodal displacements are contained in vector $\mathbf{q}={ }^{t}\left\{v_{1}^{k}, v_{2}^{k}, v_{3}^{k}, w_{1}^{k}, w_{2}^{k}, w_{3}^{k}, \cdots \times 8\right\}(k=1, \ldots, 8)$ and interpolated to integration points with the help of shape functions $\mathbf{N}$, leading to element displacement vector $\mathbf{u}={ }^{t}\left\{v_{1}, v_{2}, v_{3}, w_{1}, w_{2}, w_{3}\right\}$ :

$$
\mathbf{u}=\left\{\begin{array}{c}
\mathbf{v} \\
\mathbf{w}
\end{array}\right\}=\mathbf{N} \cdot \mathbf{q}=\mathbf{N}\left\{\begin{array}{c}
v^{k} \\
w^{k}
\end{array}\right\}_{(k=1, \ldots, 8)} .
$$

Its derivative writes

$$
\boldsymbol{\theta}(\mathbf{u})=\left\{\begin{array}{c}
v_{i, j} \\
w_{i, j}
\end{array}\right\}=\left[\begin{array}{c}
\mathbf{G}_{1} \\
\mathbf{G}_{2}
\end{array}\right]\left\{\begin{array}{c}
v^{k} \\
w^{k}
\end{array}\right\}_{(k=1, \ldots, 8)},
$$

in which ${ }^{t}\left[\mathbf{G}_{1}, \mathbf{G}_{2}\right]$ is denoted as $\mathbf{G}$ and called gradient matrix. Expressions of $\mathbf{G}_{1}$ and $\mathbf{G}_{2}$ are given by Equations (A.30) and (A.31) in Appendix B.

Both $\mathbf{E}^{(0)}(\mathbf{u})$ and $\mathbf{E}^{(1)}(\mathbf{u})$ are composed of the linear $\mathbf{E}_{l}^{(i)}(i=1,2)$ and the nonlinear $\mathbf{E}_{n l}^{(i)}$, which means $\mathbf{E}^{(i)}=\mathbf{E}_{l}^{(i)}+\mathbf{E}_{n l}^{(i)}$. They are expressed as function of the covariant base vectors $\mathbf{a}_{i}$ and displacement gradient vector $\theta(u)$ :

$$
\mathbf{E}_{l}=\left\{\begin{array}{l}
\mathbf{E}_{l}^{(0)} \\
\mathbf{E}_{l}^{(1)}
\end{array}\right\}=\mathbf{R} \cdot \boldsymbol{\theta}(\mathbf{u}),
$$

in which $\mathbf{R}$ refers to the matrix given by Equation (A.32) in Appendix B. Similarly, vector $\mathbf{E}_{n l}$ can be calculated by

$$
\mathbf{E}_{n l}=\left\{\begin{array}{l}
\mathbf{E}_{n l}^{(0)} \\
\mathbf{E}_{n l}^{(1)}
\end{array}\right\}=\frac{1}{2} \mathbf{A} \cdot \boldsymbol{\theta}(\mathbf{u}),
$$

in which $\mathbf{A}$ is the matrix given by Equation (A.33) in Appendix B. Now with $\mathbf{E}_{l}$ (Equation (A.15)) and $\mathbf{E}_{n l}$ (Equation (A.16)) discretized, the compatible strain $\mathbf{E}^{u}$ can be calculated by

$$
\mathbf{E}^{u}=\mathbf{E}_{l}+\mathbf{E}_{n l}=\left(\mathbf{R}+\frac{1}{2} \mathbf{A}\right) \boldsymbol{\theta}(\mathbf{u}) .
$$

We can verify that the variational form of $\mathbf{E}^{u}$ writes

$$
\delta \mathbf{E}^{u}=(\mathbf{R}+\mathbf{A}) \boldsymbol{\theta}(\mathbf{u})=(\mathbf{R}+\mathbf{A}) \mathbf{G} \delta \mathbf{q},
$$

where $\mathbf{G}$ denotes the gradient matrix defined in Equation (A.14), and $\delta \mathbf{q}$ the variational form of nodal displacements. We introduce $\mathbf{B}$ to denote the matrix $(\mathbf{R}+\mathbf{A}) \mathbf{G}$, then

$$
\delta \mathbf{E}^{u}=\overline{\mathbf{B}} \delta \mathbf{q} .
$$

Like in the work of Bischoff [24], the proposed shell can be formulated using generalized stress measures, composed of $\mathbf{n}$ and $\mathbf{m}$. They are energetically conjugated to their strain counterparts $\mathbf{E}^{(0)}$ and $\mathbf{E}^{(1)}$ and are computed by integrating the stress and moment through the shell thickness. Both $\mathbf{n}$ and $\mathbf{m}$ are arranged in vector $\mathbf{S}={ }^{t}\left\{{ }^{t} \mathbf{n},{ }^{t} \mathbf{m}\right\}$, in which

$$
\begin{aligned}
& \mathbf{n}={ }^{t}\left\{n^{11}, n^{22}, n^{33}, n^{12}, n^{13}, n^{23}\right\} \\
& \mathbf{m}={ }^{t}\left\{m^{11}, m^{22}, m^{33}, m^{12}, m^{13}, m^{23}\right\}
\end{aligned}
$$


A.2.1. Discretization of the enhanced assumed strain $\tilde{\mathbf{E}}$. The present shell model is conceived on the basis of a multifield strain formulation. The compatible strain field derived from displacements is extended by a linear term which acts in the shell transversal direction, leading to the seventh degree of freedom. This linear strain field $\tilde{\mathbf{E}}$, namely assumed strain, is discretized on element level. It does not require interelement continuity, neither contribute to the total number of nodal degrees of freedom. Equation (A.5) defines the linear assumed strain, which here is discretized by the bi-linear form

$$
\tilde{E}_{33}=\alpha_{1}+\alpha_{2} \theta_{1}+\alpha_{3} \theta_{2}+\alpha_{4} \theta_{1} \theta_{2},
$$

or in the following form:

$$
\tilde{\mathbf{E}}=\mathbf{B}_{\alpha} \cdot \boldsymbol{\alpha},
$$

where $\mathbf{B}_{\alpha}$ is given by Equation (A.35) in Appendix B. Here, $\mathbf{B}_{\alpha}$ is constructed with local curvilinear coordinates $\left(\theta^{1}, \theta^{2}\right)$ of the current integration point. So $\tilde{\mathbf{E}}$ is determined (Equation (A.22)) based on $\boldsymbol{\alpha}$, which is an internal variable evolving independently within each element. We detail in the following the determination of $\boldsymbol{\alpha}$, thus the assumed strain $\tilde{\mathbf{E}}$.

A.2.2. Discretization and resolution of the problem governing equations. Let us recall the shell problem definition (Equation (A.12)). By using previously defined $\mathbf{B}_{\alpha}$ (Equation (A.22)) and $\overline{\mathbf{B}}$ (Equation (A.19)), governing equations of the problem (Equation (A.12)) can be written as

$$
\left\{\begin{array}{l}
\left.\int_{\Omega_{e}}{ }^{t} \overline{\mathbf{B}} \cdot \mathbf{S} \mathrm{d} \Omega-\lambda \mathbf{F}_{\text {ext }}(\delta \mathbf{u})\right\}=\mathbf{0} \\
\int_{\Omega_{e}}{ }^{t} \mathbf{B}_{\alpha} \cdot \mathbf{S} \mathrm{d} \Omega=\mathbf{0} .
\end{array}\right.
$$

We identify in the first equation of Equation (A.23) the elementary internal force $\mathbf{F}_{\text {int }}$, and from second equation of Equation (A.23) the relative residual with respect to the compatibility condition. Hence,

$$
\left\{\begin{array}{l}
\mathbf{F}_{\mathrm{int}}=\int_{\Omega_{e}}{ }^{t} \overline{\mathbf{B}} \cdot \mathbf{S} \mathrm{d} \Omega \\
\boldsymbol{R e}=\int_{\Omega_{e}}{ }^{t} \mathbf{B}_{\alpha} \cdot \mathbf{S} \mathrm{d} \Omega .
\end{array}\right.
$$

Its variational form writes

$$
\left\{\begin{array}{l}
\delta \mathbf{F}_{\text {int }}=\mathbf{K}_{u u} \cdot \delta \mathbf{q}+\mathbf{K}_{u \alpha} \cdot \delta \boldsymbol{\alpha} \\
\delta \mathbf{R e}=\mathbf{K}_{\alpha u} \cdot \delta \mathbf{q}+\mathbf{K}_{\alpha \alpha} \cdot \delta \boldsymbol{\alpha},
\end{array}\right.
$$

where the matrices involved refer to

$$
\begin{aligned}
\mathbf{K}_{u u} & =\int_{\Omega_{e}}\left({ }^{t} \mathbf{G} \cdot \mathbf{M} \cdot \mathbf{G}+{ }^{t} \overline{\mathbf{B}} \cdot \mathbf{C} \cdot \overline{\mathbf{B}}\right) \mathrm{d} \Omega, \\
\mathbf{K}_{\alpha u} & =\int_{\Omega_{e}}{ }^{t} \mathbf{B}_{\alpha} \cdot \mathbf{C} \cdot \overline{\mathbf{B}} \mathrm{d} \Omega, \\
\mathbf{K}_{u \alpha} & =\int_{\Omega_{e}}{ }^{t} \overline{\mathbf{B}} \cdot \mathbf{C} \cdot \mathbf{B}_{\alpha} \mathrm{d} \Omega={ }^{t} \mathbf{K}_{\alpha u}, \\
\mathbf{K}_{\alpha \alpha} & =\int_{\Omega_{e}}{ }^{t} \mathbf{B}_{\alpha} \cdot \mathbf{C} \cdot \mathbf{B}_{\alpha} \mathrm{d} \Omega .
\end{aligned}
$$


Matrix form of $\mathbf{M}$ in the first equation of Equation (A.26) is defined with respect to the initial state of stress. For exact form of M, please refer to Equation (A.34) in Appendix B. We then prescribe based on Equations (A.24) and (A.25) an iterative procedure to solve the shell problem, so

$$
\left\{\begin{array}{l}
\bigwedge_{e=1}^{n E l m}\left(\mathbf{F}_{\mathrm{int}}+\mathbf{K}_{u u} \cdot \delta \mathbf{q}+\mathbf{K}_{u \alpha} \cdot \delta \boldsymbol{\alpha}-\mathbf{F}_{\mathrm{ext}}-\delta \mathbf{F}_{\mathrm{int}}\right)_{e}=\mathbf{0} \\
\bigwedge_{e=1}^{n E l m}\left(\mathbf{R e}+\mathbf{K}_{\alpha u} \cdot \delta \mathbf{q}+\mathbf{K}_{\alpha \alpha} \cdot \delta \boldsymbol{\alpha}\right)_{e}=\mathbf{0}
\end{array}\right.
$$

where $\bigwedge_{e=1}^{n E l m}$ refers to classical finite element assembly operator, adding elementary force or stiffness contributions into their global vector or matrix counterparts. Condensation of the two equations in Equation (A.27) eliminates $\delta \boldsymbol{\alpha}$, leading to the following iterative tangent problem written as function of displacements:

$$
\mathbf{K}_{\mathbf{T}} \delta \mathbf{q}=\mathbf{F}_{\mathbf{R}},
$$

in which $\mathbf{K}_{\mathbf{T}}$ and $\mathbf{F}_{\mathbf{R}}$ denote

$$
\left\{\begin{array}{l}
\mathbf{K}_{\mathbf{T}}=\bigwedge_{e=1}^{n E l m}\left(\mathbf{K}_{u u}-\mathbf{K}_{u \alpha} \cdot \mathbf{K}_{\alpha \alpha}^{-1} \cdot \mathbf{K}_{\alpha u}\right)_{e} \\
\mathbf{F}_{\mathbf{R}}=\bigwedge_{e=1}^{n E l m}\left(\mathbf{F}_{\mathrm{ext}}-\mathbf{F}_{\mathrm{int}}+\mathbf{K}_{u \alpha} \cdot \mathbf{K}_{\alpha \alpha}^{-1} \cdot \mathbf{R e}\right)_{e}
\end{array}\right.
$$

\section{APPENDIX B}

\section{B.1. Matrix representations used in the shell element}

We provide here in detail the matrices used in the shell element discretization presented in Appendix A.

The gradient operator $\mathbf{G}$ writes ${ }^{t}\left[\mathbf{G}_{1}, \mathbf{G}_{2}\right]$ in which $\mathbf{G}_{1}$ can be found in the following matrix expression:

$$
\left\{v_{i, j}\right\}=\left\{\begin{array}{c}
v_{1,1} \\
v_{1,2} \\
w_{1} \\
v_{2,1} \\
v_{2,2} \\
w_{2} \\
v_{3,1} \\
v_{3,2} \\
w_{3}
\end{array}\right\}=\left[\begin{array}{cccccc:c}
N_{, 1}^{k} & 0 & 0 & 0 & 0 & 0 & \\
N_{, 2}^{k} & 0 & 0 & 0 & 0 & 0 & \\
0 & 0 & 0 & N^{k} & 0 & 0 & \\
0 & N_{, 1}^{k} & 0 & 0 & 0 & 0 & \\
0 & N_{, 2}^{k} & 0 & 0 & 0 & 0 & \cdots \times 8 \\
0 & 0 & 0 & 0 & N^{k} & 0 & \\
0 & 0 & N_{, 1}^{k} & 0 & 0 & 0 & \\
0 & 0 & N_{, 2}^{k} & 0 & 0 & 0 & \\
0 & 0 & 0 & 0 & 0 & N^{k}
\end{array}\right]\left\{\begin{array}{c}
v_{1}^{k} \\
v_{2}^{k} \\
v_{3}^{k} \\
w_{1}^{k} \\
w_{2}^{k} \\
w_{3}^{k} \\
\vdots \\
\times 8
\end{array}\right\} ;
$$


and for $\mathbf{G}_{2}$,

$$
\left\{w_{i, j}\right\}=\left\{\begin{array}{l}
w_{1,1} \\
w_{1,2} \\
w_{1,3} \\
w_{2,1} \\
w_{2,2} \\
w_{2,3} \\
w_{3,1} \\
w_{3,2} \\
w_{3,3}
\end{array}\right\}=\left[\begin{array}{cccccc:c}
0 & 0 & 0 & N_{, 1}^{k} & 0 & 0 & \\
0 & 0 & 0 & N_{, 2}^{k} & 0 & 0 & \\
0 & 0 & 0 & 0 & 0 & 0 & \\
0 & 0 & 0 & 0 & N_{, 1}^{k} & 0 & \\
0 & 0 & 0 & 0 & N_{, 2}^{k} & 0 & \ldots \times 8 \\
0 & 0 & 0 & 0 & 0 & 0 & \\
0 & 0 & 0 & 0 & 0 & N_{, 1}^{k} \\
0 & 0 & 0 & 0 & 0 & N_{, 2}^{k} \\
0 & 0 & 0 & 0 & 0 & 0 &
\end{array}\right]\left\{\begin{array}{c}
v_{1}^{k} \\
v_{2}^{k} \\
v_{3}^{k} \\
w_{1}^{k} \\
w_{2}^{k} \\
w_{3}^{k} \\
\vdots \\
\times 8
\end{array}\right\} .
$$

Then, $\mathbf{R}$ is found in the following matrix expression:

$$
\left\{\begin{array}{c}
E_{l 11}^{(0)} \\
E_{l 22}^{(0)} \\
E_{l 33}^{(0)} \\
2 E_{l 12}^{(0)} \\
2 E_{l 13}^{(0)} \\
2 E_{l 23}^{(0)} \\
E_{l 11}^{(1)} \\
E_{l 22}^{(1)} \\
E_{l 33}^{(1)} \\
2 E_{l 12}^{(1)} \\
2 E_{l 13}^{(1)} \\
2 E_{l 23}^{(1)}
\end{array}\right\}=\left[\begin{array}{ccccccccccccc}
a_{1}^{1} & 0 & 0 & a_{1}^{2} & 0 & 0 & a_{1}^{3} & 0 & 0 & \\
0 & a_{2}^{1} & 0 & 0 & a_{2}^{2} & 0 & 0 & a_{2}^{3} & 0 & & \\
0 & 0 & a_{3}^{1} & 0 & 0 & a_{3}^{2} & 0 & 0 & a_{3}^{3} & & \\
a_{2}^{1} & a_{1}^{1} & 0 & a_{2}^{2} & a_{1}^{2} & 0 & a_{2}^{3} & a_{1}^{3} & 0 & & & \\
a_{3}^{1} & 0 & a_{1}^{1} & a_{3}^{2} & 0 & a_{1}^{2} & a_{3}^{3} & 0 & a_{1}^{3} & & \\
0 & a_{3}^{1} & a_{2}^{1} & 0 & a_{3}^{2} & a_{2}^{2} & 0 & a_{3}^{3} & a_{2}^{3} & & & \\
0 & 0 & 0 & 0 & 0 & 0 & 0 & 0 & 0 & & & \\
0 & 0 & 0 & 0 & 0 & 0 & 0 & 0 & 0 & & & \\
0 & 0 & 0 & 0 & 0 & 0 & 0 & 0 & 0 & & & \\
0 & 0 & 0 & 0 & 0 & 0 & 0 & 0 & 0 & & & \\
0 & 0 & 0 & 0 & 0 & 0 & 0 & 0 & 0 & & & \\
0 & 0 & 0 & 0 & 0 & 0 & 0 & 0 & 0 & & & \\
v_{1,2} \\
w_{1,1} \\
v_{2,1} \\
v_{2,2} \\
w_{2} \\
v_{3,1} \\
v_{3,2} \\
w_{3} \\
w_{1,1} \\
w_{1,2} \\
w_{1,3} \\
w_{2,1} \\
w_{2,2} \\
w_{2,3} \\
w_{3,1} \\
w_{3,2} \\
w_{3,3}
\end{array}\right\} .
$$


And $\mathbf{A}$ writes in the following form:

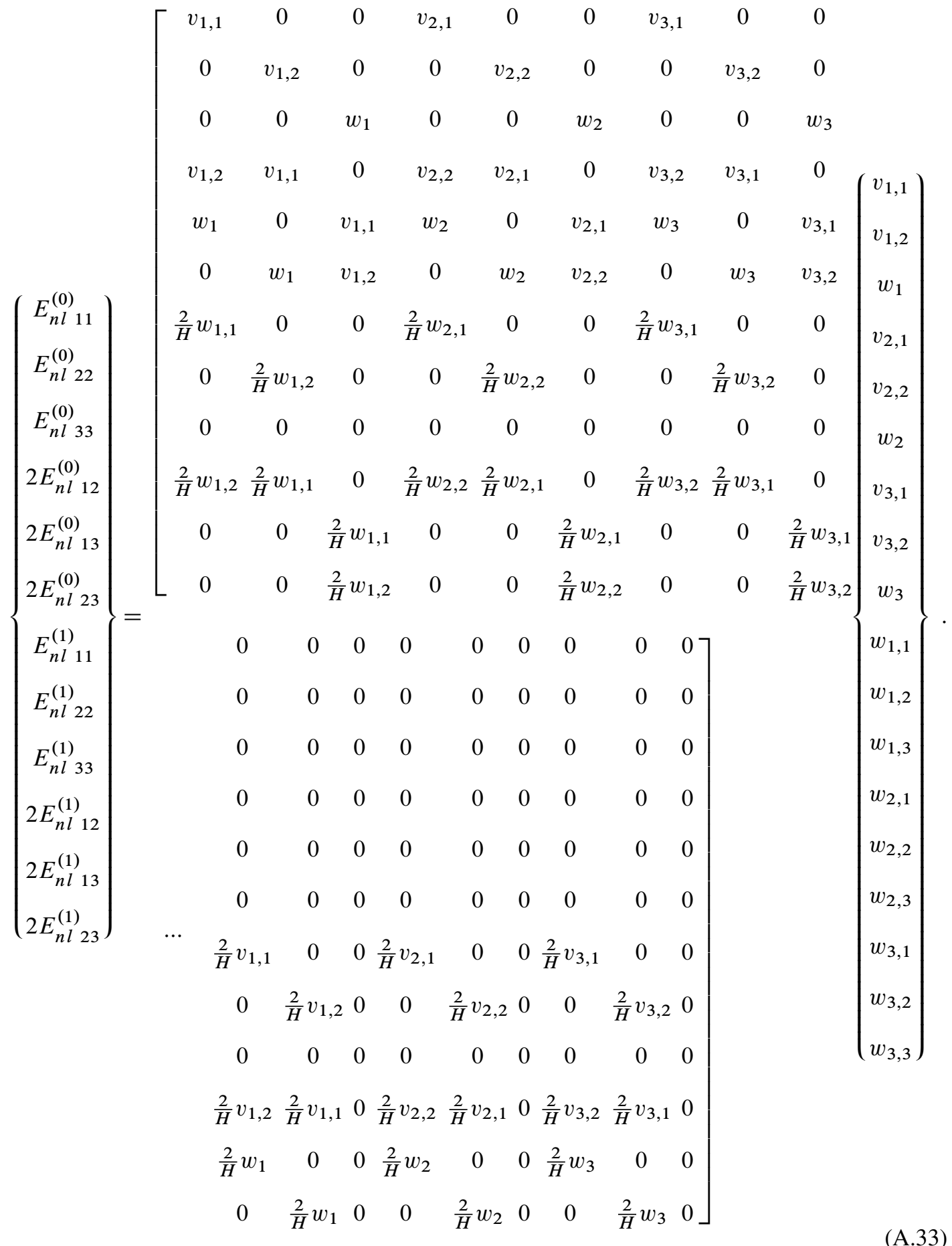


$\mathbf{M}$ is found in the following:

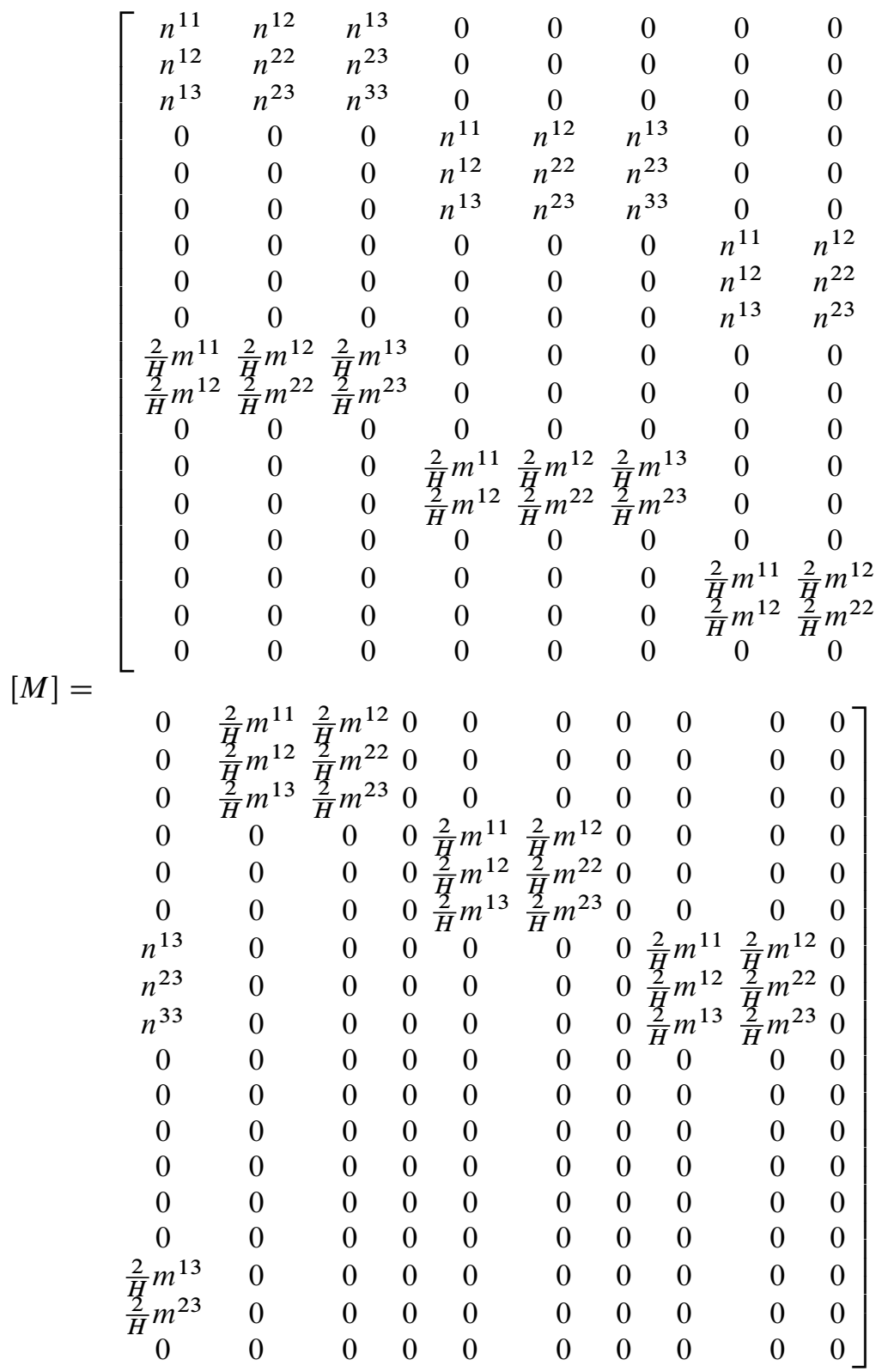

$\mathbf{B}_{\alpha}$ is found in the following expression:

$$
\{\tilde{E}\}=\left\{\begin{array}{c}
0 \\
0 \\
0 \\
0 \\
0 \\
0 \\
0 \\
0 \\
\tilde{E}_{33} \\
0 \\
0 \\
0
\end{array}\right\}=\frac{2}{H}\left[\begin{array}{cccc}
0 & 0 & 0 & 0 \\
0 & 0 & 0 & 0 \\
0 & 0 & 0 & 0 \\
0 & 0 & 0 & 0 \\
0 & 0 & 0 & 0 \\
0 & 0 & 0 & 0 \\
0 & 0 & 0 & 0 \\
0 & 0 & 0 & 0 \\
1 & \theta^{1} & \theta^{2} & \theta^{1} \theta^{2} \\
0 & 0 & 0 & 0 \\
0 & 0 & 0 & 0 \\
0 & 0 & 0 & 0
\end{array}\right]\left\{\begin{array}{c}
\alpha_{1} \\
\alpha_{2} \\
\alpha_{3} \\
\alpha_{4}
\end{array}\right\} .
$$




\section{ACKNOWLEDGEMENTS}

We gratefully acknowledge the support this work enjoys from the Institut Universitaire de France (IUF), and from the French state program 'Investment in the future' operated by the National Research Agency (ANR) under the reference ANR-11-LABX-0008-01 (LabEx DAMAS).

\section{REFERENCES}

1. Reddy J. On refined computational models of composite laminates. International Journal for Numerical Methods in Engineering 1989; 27:361-382.

2. Noor A, Burton W. Assessment of shear deformation for multilayered composite plates. Applied Mechanics Reviews 1989; 42:1-12.

3. Reddy J. An evaluation of equivalent-single-layer and layerwise theories of composite laminates. Composite Structures 1993; 25(1):21-35.

4. Vu-Quoc L, Ebcioglu I. Multilayer shells: geometrically-exact formulation of equations of motion. International Journal of Solids and Structures 2000; 37:6705-6737.

5. Ugrimov S. Generalized theory of multilayer plates. International Journal of Solids and Structures 2002; 39(4): 819-839.

6. Abbès B, Guo Y. Analytic homogenization for torsion of orthotropic sandwich plates: application to corrugated cardboard. Composite Structures; 92:699-706.

7. Talbi N, Batti A, Ayad R, Guo Y. An analytical homogenization model for finite element modelling of corrugated cardboard. Composite Structures 2009; 88:280-289.

8. Lebée A, Sab K. Transverse shear stiffness of a chevron folded core used in sandwich construction. International Journal of Solids and Structures 2010; 47:2620-2629.

9. Lebée A, Sab K. A bending-gradient model for thick plates. Part I: theory. International Journal of Solids and Structures 2011; 48:2878-2888.

10. Lebée A, Sab K. A bending-gradient model for thick plates, part II: closed-form solutions for cylindrical bending of laminates. International Journal of Solids and Structures 2011; 48:2889-2901.

11. Cartraud P, Messager T. Computational homogenization of periodic beam-like structures. International Journal of Solids and Structures 2006; 43(3-4):686-696.

12. Buannic N, Cartraud P, Quesnel T. Homogenization of corrugated core sandwich panels. Composite Structures 2003; 59:299-312

13. Feyel $\mathrm{F}$, Chaboche J. $\mathrm{FE}^{2}$ multiscale approach for modeling the elastoviscoplastic behavior of long fiber $\mathrm{SiC} / \mathrm{Ti}$ composite materials. Computer Methods in Applied Mechanics and Engineering 2000; 183:309-330.

14. Terada K, Kikuchi N. A class of general algorithms for multi-scale analysis of heterogeneous media. Computer Methods in Applied Mechanics and Engineering 2001; 190:5427-5464.

15. Kousnetzova VG, Brekelmans WAM, Baaijens F. An approach to micro-macro modeling of heterogeneous materials. Computational Mechanics 2001; 27:37-48.

16. Miehe C, Schröder J, Becker M. Computational homogenization analysis in finite elasticity: material and structural instabilities on the micro- and macro-scales of periodic composites and their interactions. Computer Methods in Applied Mechanics and Engineering 2002; 191(4):4971-5005.

17. Miehe C. Computational micro-to-macro transitions for discretized micro-structures of heterogeneous materials at finite strains based on the minimization of averaged incremental energy. Computer Methods in Applied Mechanics and Engineering 2003; 192:559-591.

18. Nezamabadi S, Yvonnet J, Zahrouni H, Potier-Ferry M. A multilevel computational strategy for handling microscopic and macroscopic instabilities. Computer Methods in Applied Mechanics and Engineering 2009; 198:2099-2110.

19. Coenen EWC, Kouznetsova VG, Geers MGD. Computational homogenization for heterogeneous thin sheets. International Journal for Numerical Methods in Engineering 2010; 83:1180-1205.

20. Helfen C, Diebels S. A numerical homogenization method for sandwich plates based on a plate theory with thickness change. Zamm-Zeitschrift fur Angewandte Mathematik und Mechanik 2013; 93:113-125.

21. Braun M, Bischoff M, Ramm E. Nonlinear shell formulations for complete three-dimensional constitutive laws including composites and laminates. Computational Mechanics 1994; 15:1-18.

22. Buchter N, Ramm E, Roehl D. Three-dimensional extension of nonlinear shell formulation based onthe enhanced assumed strain concept. International Journal for Numerical Methods in Engineering 1994; 37:2551-2568.

23. Sansour C. A theory and finite element formulation of shells at finite deformations involving thickness change: circumventing the use of a rotation tensor. Archive of Applied Mechanics 1995; 65:194-216.

24. Bischoff M, Ramm E. On the physical significance of higher order kinematic and static variables in a threedimensional shell formulation. International Journal of Solids and Structures 2000; 37:6933-6960.

25. ABAQUS TM. Version 6.8. Karlsson and Sorensen Inc: Hibbit, 2008.

26. Riks E. The application of Newton's method to the problem of elastic stability. Journal of Applied Mechanics 1972; 39:1060-1065. 
27. Riks E. An incremental approach to the solution of snapping and buckling problems. International Journal of Solids and Structures 1979; 15:529-551.

28. Ben Dhia H. Multiscale mechanical problems : the Arlequin method. Comptes Rendus Mathématique, Académie des Sciences, Paris IIb 1998; 326:899-904.

29. Simo JC, Rifai MS, Fox DD. On a stress resultant geometrically exact shell model. Part IV: variable thickness shells with through-the-thickness stretching. Computer Methods in Applied Mechanics and Engineering 1990; 81(1): 91-126. 